\title{
A YOUNG MEASURES APPROACH TO QUASISTATIC EVOLUTION FOR A CLASS OF MATERIAL MODELS WITH NONCONVEX ELASTIC ENERGIES *
}

\author{
Alice Fiaschi ${ }^{1}$
}

\begin{abstract}
Rate-independent evolution for material models with nonconvex elastic energies is studied without any spatial regularization of the inner variable; due to lack of convexity, the model is developed in the framework of Young measures. An existence result for the quasistatic evolution is obtained in terms of compatible systems of Young measures. We also show as this result can be equivalently reformulated with probabilistic language and leads to the description of the quasistatic evolution in terms of stochastic processes on a suitable probability space.
\end{abstract}

Mathematics Subject Classification. 74B20, 28A33, 74G65, 49J45.

Received November 7, 2007.

Published online April 26, 2008.

\section{INTRODUCTION}

The energetic formulation of many rate-independent evolution processes for elastic materials involve an energy functional depending on the elastic deformation of the body and on an internal variable; an important role is played by the dissipation distance, which depends just on the internal variable (see e.g. $[9,14,15])$.

The standard method to solve this kind of problems is via time-discretization and resolution of incremental minimum problems (see [11-13,16] and references therein); to apply this method, lower semicontinuity and coerciveness of the energy functional are needed in the space where the problem is formulated. form

As in [8], we consider the case in which the energy functional $\mathcal{W}$ and the dissipation distance $\mathcal{H}$ take the

$$
\begin{gathered}
\mathcal{W}(z, v)=\int_{D} W(z(x), \nabla v(x)) \mathrm{d} x, \\
\mathcal{H}\left(z_{1}-z_{0}\right)=\int_{D} H\left(z_{1}(x)-z_{0}(x)\right) \mathrm{d} x,
\end{gathered}
$$

where $D \subset \mathbb{R}^{d}$ is the reference configuration.

Keywords and phrases. Quasistatic evolution, rate-independent processes, elastic materials, incremental problems, Young measures.

* This work is part of the Project "Calculus of Variations" 2006, supported by the Italian Ministry of University and Research.

1 SISSA, via Beirut 2-4, 34014 Trieste, Italy; fiaschi@sissa.it 
Since for the mechanical applications considered in [8] it is not natural to assume $\mathcal{W}$ convex with respect to the internal variable $z$, there is no function space where the energy functional can be simultaneously lower semicontinuous and coercive. The way proposed in [8] to overcome this difficulty is to assume quasiconvexity of $W$ with respect to its second argument and to add a spatial regularizing term of the form

$$
\eta\|\nabla z\|_{2}^{2}
$$

with $\eta>0$ : thanks to the hypotheses made on $W$ and $H$, the regularized functional is coercive in suitable Sobolev spaces, and classical results (see [1]) guarantees its lower semicontinuity in the same spaces; however the regularizing term (1.3) is not always physically justified.

In this paper we propose an approach to this problem which does not require any assumption on the convexity/quasiconvexity of the energy functional and does not need any regularizing term: lower semicontinuity and coerciveness are obtained by formulating the problem in a suitable space of Young measures, as proposed in [6] for a different problem.

Without entering in the details of the hypotheses, we just mention that we will assume that the body is subjected to an external load $\boldsymbol{l}(t)$ depending on time and to a time-dependent prescribed boundary deformation $\varphi(t)$; moreover we will ask that $W: \mathbb{R}^{m} \times \mathbb{R}^{N \times d} \rightarrow[0,+\infty)$ has quadratic growth and that $H: \mathbb{R}^{m} \rightarrow[0,+\infty)$ is convex and has linear growth.

Given an initial value of the variables $\left(z_{0}, v_{0}\right)$ and a partition of the time interval $[0, T]$ in which we study the evolution

$$
0=t^{0}<t^{1}<\ldots<t^{k}=T
$$

the approximate solution should be defined inductively by solving the following incremental minimum problem:

$$
\inf \left\{\mathcal{W}(z, v)-\left\langle\boldsymbol{l}\left(t^{i}\right), v\right\rangle+\mathcal{H}\left(z-z\left(t^{i-1}\right)\right)\right\}
$$

among all $(z, v)$ which make the energy finite and satisfy the boundary condition at time $t^{i}$. As anticipated, these problems are not well-posed in Sobolev spaces and we will present an explicit example in which (1.4) has actually no solution (see Rem. 7.3).

To obtain lower semicontinuity and coerciveness of the energy functional we place the problem in a suitable space of Young measures and solve the incremental minimum problems in this extended setting. Differently from [6], the quadratic growth hypothesis on $W$ allows us to use Young measures with finite second moments instead of generalized Young measures; to this aim we specialize to the more regular setting the definition and properties of the space of compatible systems introduced in [5] .

The next step is the study of the convergence of the approximate solutions as the time step $t^{i}-t^{i-1}$ tends to 0 . Up to careful choices of subsequences, we can obtain the convergence of the approximations to a pair $(\boldsymbol{\nu}, \boldsymbol{\mu})$, with $\boldsymbol{\nu}$ a time-dependent family of Young measures with finite second moments and values in $\mathbb{R}^{m} \times \mathbb{R}^{N \times d}$, and $\boldsymbol{\mu}$ a compatible system of Young measures with finite second moments and values in $\mathbb{R}^{m}$, connected to $\boldsymbol{\nu}$ by a suitable projection property.

The main result is Theorem 6.15 , which shows that this pair satisfies a global stability condition and an energy inequality, suitably reformulated in Young measures language (see Def. 6.14); therefore it can be considered as a solution of the quasistatic evolution problem in the framework of Young measures.

Since we do not need to use generalized Young measures, we can rephrase the definition of compatible systems introduced in [6] using a probabilistic language: in Theorem 3.3, with a modified version of Kolmogorov Theorem, we prove that we can associate to any compatible system of Young measures with finite second moments a suitable stochastic process on a probability space of the form $(D \times \Omega, P)$.

The advantage of the probabilistic formulation is that it motivates, in some sense, and clarifies the notions related to compatible systems of Young measures: in particular the notion of variation of a compatible system in a time interval $[a, b]$ agrees with the usual variation on $[a, b]$ of the corresponding stochastic process $\left(\boldsymbol{Z}_{t}\right)_{t \in[0, T]}$ 
considered as function from $[0, T]$ into $\mathrm{L}^{1}\left(D \times \Omega ; \mathbb{R}^{m}\right)$. Moreover, thanks to Theorem 3.5, the pair $(\boldsymbol{\nu}, \boldsymbol{\mu})$ representing the solution can be described by a unique stochastic process with values in $\mathbb{R}^{m} \times \mathbb{R}^{N \times d}$.

In the last section we give an alternative proof of the main theorem under the special assumption that the stored energy density is quasiconvex with respect to its second argument; we use the result in [8], Section 4, to obtain solutions of spatially regularized problems, and prove that we can pass to the limit as the regularization parameter vanishes, to obtain a globally stable quasistatic evolution in terms of Young measures in the sense of Definition 6.14.

\section{MATHEMATICAL PRELIMINARIES}

Functions and measures. We denote by $\mathcal{L}^{d}$ the Lebesgue measure on $\mathbb{R}^{d}, d \geq 1$, and by $\mathcal{H}^{k}$ the $k$ dimensional Hausdorff measure. $\|\cdot\|_{p}$ is the usual norm on $\mathrm{L}^{p} \cdot \mathrm{H}^{1}\left(D ; \mathbb{R}^{N}\right)$ denotes the usual Sobolev space of all functions from an open domain $D \subseteq \mathbb{R}^{d}$ into $\mathbb{R}^{N}$ lying in $\mathrm{L}^{2}$ with their first derivatives. The symbol $\langle\cdot, \cdot\rangle$ will denote a duality pairing depending on the context.

Given a finite dimensional Hilbert space $\Xi, f: D \times \Xi \rightarrow \mathbb{R}$ is said to be a Carathéodory function if $f(x, \cdot)$ is continuous for a.e. $x \in D$ and $f(\cdot, \xi)$ is measurable for every $\xi \in \Xi$.

Given $D$ an open bounded domain in $\mathbb{R}^{d}$ and $\Xi$ a finite dimensional Hilbert space, we denote by $M_{b}(D \times \Xi)$ the space of all bounded Radon measures on $D \times \Xi$; this space can be identified with the dual of the Banach space $\mathrm{C}_{0}(\mathrm{D} \times \Xi)$ of all continuous functions $\phi: D \times \Xi \rightarrow \mathbb{R}$ such that $|\phi| \geq \varepsilon$ is compact for every $\varepsilon>0$. We will consider on $M_{b}(D \times \Xi)$ the weak* topology deriving from this duality.

Let $\nu$ and $\mu$ be nonnegative measures in $M_{b}(D)$ and $M_{b}(D \times \Xi)$, respectively, such that $\pi_{D}(\mu)=\nu$. The Disintegration Theorem (see, e.g., [18], Appendix A2) guarantees the existence of a measurable family $\left(\mu^{x}\right)_{x \in D}$ of probability measures on $\Xi$, such that

$$
\int_{D \times \Xi} f(x, \xi) \mathrm{d} \mu(x, \xi)=\int_{D}\left(\int_{\Xi} f(x, \xi) \mathrm{d} \mu^{x}(\xi)\right) \mathrm{d} \nu(x),
$$

for every bounded Borel function $f: D \times \Xi \rightarrow \mathbb{R}$. The measures $\mu^{x}$ are uniquely determined for a.e. $x \in D$ and we will write

$$
\mu=\int_{D} \mu^{x} \mathrm{~d} \nu(x)
$$

In the whole paper we will use the following notation: $\pi_{D}$ and $\pi_{\Xi}$ will denote the usual projections of the product space $D \times \Xi$ on $D$ and $\Xi$ respectively; in the case $\Xi=\Xi_{1} \times \Xi_{2}, \tilde{\pi}_{\Xi_{i}}$ will denote the projection of $D \times \Xi_{1} \times \Xi_{2}$ on $D \times \Xi_{i}$ and $\pi_{\Xi_{i}}$ the projection of $\Xi_{1} \times \Xi_{2}$ on $\Xi_{i}$, for $i=1,2$.

Young measures. Now we will recall the definition and the main properties of Young measures.

In the whole paper $D$ will denote a bounded open domain in $\mathbb{R}^{d}$ and $\Xi, \Xi_{1}, \Xi_{2}$ will denote finite dimensional Hilbert spaces; we will assume, without loss of generality, that

$$
\mathcal{L}^{d}(D)=1 \text {. }
$$

The space $Y(D ; \Xi)$ of the Young measures on $D$ with values in $\Xi$ is the space of all nonnegative measures $\mu \in M_{b}(D \times \Xi)$ such that

$$
\pi_{D}(\mu)=\mathcal{L}^{d}
$$

Applying the Disintegration Theorem to $\mu \in Y(D ; \Xi)$, we deduce the existence of a measurable family of probability measures on $\Xi,\left(\mu^{x}\right)_{x \in D}$, with

$$
\mu=\int_{D} \mu^{x} \mathrm{~d} x .
$$


$Y^{2}(D ; \Xi)$ denotes the space of all $\mu \in Y(D ; \Xi)$, whose second moment

$$
\int_{D \times \Xi}|\xi|^{2} \mathrm{~d} \mu(x, \xi)=\int_{D}\left(\int_{\Xi}|\xi|^{2} \mathrm{~d} \mu^{x}(\xi)\right) \mathrm{d} x
$$

is finite.

To every $u \in \mathrm{L}^{2}(D ; \Xi)$ we can associate a Young measure with finite second moment denoted by $\delta_{u}$ and defined by

$$
\int_{D \times \Xi} f(x, \xi) \mathrm{d} \delta_{u}(x, \xi):=\int_{D} f(x, u(x)) \mathrm{d} x
$$

for every bounded Borel function $f: D \times \Xi \rightarrow \mathbb{R}$. In particular for every $\bar{\xi} \in \Xi$, we will denote the Young measure associated to the function $x \mapsto \bar{\xi}$ simply by $\delta_{\bar{\xi}}$.

\section{Young measures With Probabilistic LANGUAGE}

In this section we want to point out that Young measures can be presented using a probabilistic language, and precisely the notion of random variable. While for a single Young measure this probabilistic presentation does not introduce relevant simplifications, it will be very useful in the case of families of time-dependent Young measures.

Probability spaces of the form $(D \times \Omega, \mathcal{B}(D) \otimes \mathcal{F}, P)$, where $(\Omega, \mathcal{F})$ is a measurable space, $\mathcal{B}(D)$ denotes the Borel $\sigma$-algebra on $D$, and $P$ a probability measure with the property $\pi_{D}(P)=\mathcal{L}^{d}$, will be called $\left(D, \mathcal{L}^{d}\right)$ probability spaces.

We can associate to every Young measure $\mu$ on $D$ with values in $\Xi$ a random variable $Y$ defined on a $\left(D, \mathcal{L}^{d}\right)$-probability space with values in $\Xi$, in such a way that

$$
\int_{D \times \Xi} f(x, \xi) \mathrm{d} \mu(x, \xi)=\int_{D \times \Omega} f(x, Y(x, \omega)) \mathrm{d} P(x, \omega),
$$

for every bounded Borel function $f: D \times \Xi \rightarrow \mathbb{R}$. Indeed it is enough to take as $\Omega$ the space $\Xi$ itself, as $Y$ the projection on $\Xi$, and as $P$ the measure $\mu$ itself, which is a probability measure thanks to (2.1) and (2.2). Conversely, given any $\Xi$-valued random variable $Y$ on a $\left(D, \mathcal{L}^{d}\right)$-probability space, formula $(3.1)$ defines a Young measure $\mu$, which will be denoted by $\left(\pi_{D}, Y\right)(P)$, since it coincides with the image of the measure $P$ under the map $\left(\pi_{D}, Y\right): D \times \Omega \rightarrow D \times \Xi$.

We say that a random variable $Y$ on a $\left(D, \mathcal{L}^{d}\right)$-probability space $(D \times \Omega, P)$ has finite second moment if

$$
\int_{D \times \Omega}|Y(x, \omega)|^{2} \mathrm{~d} P(x, \omega)<\infty .
$$

Hence a Young measure has finite second moment if and only if the associated random variable does.

In the particular case of $\mu=\delta_{u} \in Y^{2}(D ; \Xi)$ with $u \in \mathrm{L}^{2}(D ; \Xi)$, for every $\left(D, \mathcal{L}^{d}\right)$-probability space we can associate to $\delta_{u}$ the random variable $Y: D \times \Omega \rightarrow \Xi$, defined by $Y(x, \omega):=u(x)$ for $\mathcal{L}^{d}$-a.e. $x \in D$ and for every $\omega \in \Omega$; we will denote this random variable simply by $u$.

\subsection{Stochastic processes and compatible systems of Young measures}

As we have seen, if we deal with a single random variable the association to a Young measure is immediate; more complicated is the case of a stochastic process $\left(\boldsymbol{Y}_{t}\right)_{t \in T}$ on a $\left(D, \mathcal{L}^{d}\right)$-probability space in a time set $T$ : indeed the family of measures $\left(\left(\pi_{D}, \boldsymbol{Y}_{t}\right)(P)\right)_{t \in T}$ gives an insufficient information on the stochastic process, since in general we cannot go back to $\left(\pi_{D}, \boldsymbol{Y}_{t_{1}}, \ldots, \boldsymbol{Y}_{t_{n}}\right)(P)$, for an increasing sequence of time instants $t_{1}<\ldots<t_{n}$, just using $\left(\pi_{D}, \boldsymbol{Y}_{t_{i}}\right)(P), i=1, \ldots, n$. 
In this section, using a modification of Kolmogorov Theorem (see [10], p. 29), we want to show that the correct correspondence is between stochastic processes and compatible systems of Young measures, which have been introduced in [5] in the more general context of generalized Young measures.

Given a stochastic process $\left(\boldsymbol{Y}_{t}\right)_{t \in T}$ on a $\left(D, \mathcal{L}^{d}\right)$-probability space, with

$$
\boldsymbol{Y}_{t} \in \mathrm{L}^{2}\left(D \times \Omega ; \Xi_{t}\right)
$$

we can define a family of Young measures on $D$, indexed by the nonempty finite subsets $F$ of $T$, as

$$
\boldsymbol{\mu}_{F}:=\left(\pi_{D},\left(\boldsymbol{Y}_{t}\right)_{t \in F}\right)(P) .
$$

It is immediate to see that every $\boldsymbol{\mu}_{F}$ has finite second moment and that this family satisfies the following compatibility condition

$$
\tilde{\pi}_{G}^{F}\left(\boldsymbol{\mu}_{F}\right)=\boldsymbol{\mu}_{G},
$$

for every nonempty finite subsets $G \subset F$ of $T$, where $\tilde{\pi}_{G}^{F}: D \times \prod_{t \in F} \Xi_{t} \rightarrow D \times \prod_{s \in G} \Xi_{s} \operatorname{maps}\left(x,\left(\xi_{t}\right)_{t \in F}\right)$ in $\left(x,\left(\xi_{s}\right)_{s \in G}\right)$.

According to [5], we define a compatible system of Young measures with finite second moments on $D$ with time set $T$ and values in $\prod_{t \in T} \Xi_{t}$ as a family $\boldsymbol{\mu}=\left(\boldsymbol{\mu}_{F}\right)$ of Young measures $\boldsymbol{\mu}_{F} \in Y^{2}\left(D ; \prod_{t \in F} \Xi_{t}\right)$, with $F$ varying among all nonempty finite subsets of $T$, such that the compatibility condition (3.3) is satisfied.

The space of all such systems is denoted by $S Y^{2}\left(D ; \prod_{t \in T} \Xi_{t}\right)$; in the special case of $\Xi_{t} \equiv \Xi$, for every $t \in T$ we will use the notation $S Y^{2}(T, D ; \Xi)$.

The two following remarks are technical and will be used to prove the correspondence between compatible systems and stochastic processes.

Remark 3.1. If $\boldsymbol{\mu}$ satisfies the compatibility condition, for every nonempty finite subsets $G \subset F$ of $T$ there exists a set $N_{G}^{F}$ of $D$ with $\mathcal{L}^{d}\left(N_{G}^{F}\right)=0$, such that

$$
\pi_{G}^{F}\left(\boldsymbol{\mu}_{F}^{x}\right)=\boldsymbol{\mu}_{G}^{x} \text { for every } x \in D \backslash N_{G}^{F} .
$$

Conversely, if (3.4) holds for a.e. $x \in D$, then $\boldsymbol{\mu}$ satisfies the compatibility condition (3.3).

Hence, up to subsets of $D$ with zero measure, the compatibility condition commutes in some sense with the disintegration.

Remark 3.2. Let $Y^{2}(D ; \Xi)^{T}$ denote the set of all families of Young measures with finite second moments on $D$ with values in $\Xi$, indexed on the set $T$. Given $\left(\mu_{t}\right)_{t \in T} \in Y^{2}(D ; \Xi)^{T}$, we can always construct a compatible system $\boldsymbol{\mu} \in S Y^{2}(T, D ; \Xi)$ satisfying $\boldsymbol{\mu}_{t}=\mu_{t}$ for every $t \in T$. Indeed it is enough to define

$$
\boldsymbol{\mu}_{F}:=\int_{D}\left(\underset{t \in F}{\otimes} \mu_{t}^{x}\right) \mathrm{d} x,
$$

for every nonempty finite subset $F$ of $T$.

In the next theorem we will show that to every compatible system of Young measures with finite second moments we can associate a stochastic process on a suitable $\left(D, \mathcal{L}^{d}\right)$-probability space.

Theorem 3.3. Given a set of indices $T$ and a compatible system $\boldsymbol{\mu} \in S Y^{2}\left(D ; \prod_{t \in T} \Xi_{t}\right)$, there exist a $\left(D, \mathcal{L}^{d}\right)$ probability space and a stochastic process $\left(\boldsymbol{X}_{t}\right)_{t \in T}$ with

$$
\boldsymbol{X}_{t} \in \mathrm{L}^{2}\left(D \times \Omega ; \Xi_{t}\right)
$$


for every $t \in T$, such that

$$
\left(\pi_{D},\left(\boldsymbol{X}_{t}\right)_{t \in F}\right)(P)=\boldsymbol{\mu}_{F},
$$

for every nonempty finite subset $F$ of $T$.

Proof. Let $t_{\infty}$ be an index such that $t_{\infty} \notin T$. Set $\hat{T}:=\left\{t_{\infty}\right\} \cup T$ and let $\Xi_{t_{\infty}}:=D$.

We want to construct a system of finite dimensional distributions indexed on $\hat{T}$, satisfying the hypotheses of Kolmogorov Theorem.

Let $F$ be a nonempty finite subset of $\hat{T}$; we distinguish three cases. If $t_{\infty} \in F$ and $F \backslash\left\{t_{\infty}\right\} \neq \varnothing$ we set

$$
\nu_{F}=\boldsymbol{\mu}_{F \backslash\left\{t_{\infty}\right\}} \cdot
$$

If $t_{\infty} \notin F$, we set

$$
\boldsymbol{\nu}_{F}:=\pi \prod_{\tau \in F} \Xi_{\tau}\left(\boldsymbol{\mu}_{F}\right)
$$

where $\pi_{\prod_{\tau \in F} \Xi_{\tau}}: D \times \prod_{\tau \in F} \Xi_{\tau} \rightarrow \prod_{\tau \in F} \Xi_{\tau}$ denotes the usual projection. Finally, if $F=\left\{t_{\infty}\right\}$, we set

$$
\nu_{F}:=\mathcal{L}^{d}
$$

The system of finite dimensional distributions $\boldsymbol{\nu}$ satisfies the following compatibility condition: for every nonempty finite subsets $G \subset F$ of $\hat{T}$, we have

$$
\pi_{G}^{F}\left(\nu_{F}\right)=\nu_{G}
$$

Indeed, in the case $t_{\infty} \in F$ and $F \backslash\left\{t_{\infty}\right\} \neq \varnothing$, it follows from compatibility condition for $\boldsymbol{\mu}$ if $t_{\infty} \in G$ and $G \backslash\left\{t_{\infty}\right\} \neq \varnothing$, it comes from (2.2) if $G=\left\{t_{\infty}\right\}$, and it easily follows from (3.8) if $t_{\infty} \notin G$. In case $t_{\infty} \notin F$, (3.10) can be proved using the construction in (3.8) and the analysis of the previous case.

By (3.10), $\boldsymbol{\nu}$ satisfies the hypotheses of Kolmogorov Theorem; therefore it is enough to choose $\Omega:=\prod_{t \in T} \Xi_{t}$, $\mathcal{F}$ the product of the Borel $\sigma$-algebras of $\Xi_{t}$, for $t \in T, \boldsymbol{X}_{t}: \Omega \rightarrow \Xi_{t}$, for $t \in T$, the usual projections, and Kolmogorov Theorem guarantees the existence of a probability measure $P$ on $(D \times \Omega, \mathcal{B}(D) \times \mathcal{F})$ with $\pi_{D}(P)=\mathcal{L}^{d}$ which satisfies (3.6).

Using the previous result we will prove that we can associate to a pair of two compatible systems, connected by a further compatibility condition, a pair of stochastic processes on the same probability space.

Lemma 3.4. Let $V$ and $W$ finite dimensional Hilbert spaces, $\mu \in Y^{2}(D ; V)$ and $\nu \in Y^{2}(D ; V \times W)$ be such that

$$
\tilde{\pi}_{V}(\nu)=\mu
$$

Then for $\mathcal{L}^{d}$-a.e. $x \in D$ we have

$$
\left(\nu^{x}\right)^{v}=\nu^{(x, v)}
$$

for $\mu^{x}$-a.e. $v \in V$, where $\left(\nu^{x}\right)^{v}$ is the disintegration of $\nu^{x}$ with respect to $\mu^{x}$ and $\nu^{(x, v)}$ the disintegration of $\nu$ with respect to $\mu$.

Proof. It is easy to see, as for Remark 3.1, that there exists a set $N \subseteq D$, with $\mathcal{L}^{d}(N)=0$, such that for every $x \in D \backslash N$

$$
\pi_{V}\left(\nu^{x}\right)=\mu^{x} .
$$


Hence for every bounded Borel function $f: D \times V \times W \rightarrow \mathbb{R}$,

$$
\begin{aligned}
\int_{D \times V \times W} f(x, v, w) \mathrm{d} \nu(x, v, w) & =\int_{D}\left(\int_{V \times W} f(x, v, w) \mathrm{d} \nu^{x}(v, w)\right) \mathrm{d} x \\
& =\int_{D}\left(\int_{V}\left(\int_{W} f(x, v, w) \mathrm{d}\left(\nu^{x}\right)^{v}(w)\right) \mathrm{d} \mu^{x}(v)\right) \mathrm{d} x .
\end{aligned}
$$

On the other hand, thanks to (3.11),

$$
\begin{aligned}
\int_{D \times V \times W} f(x, v, w) \mathrm{d} \nu(x, v, w) & =\int_{D \times V}\left(\int_{W} f(x, v, w) \mathrm{d} \nu^{(x, v)}(w)\right) \mathrm{d} \mu(x, v) \\
& =\int_{D}\left(\int_{V}\left(\int_{W} f(x, v, w) \mathrm{d} \nu^{(x, v)}(w)\right) \mathrm{d} \mu^{x}(v)\right) \mathrm{d} x
\end{aligned}
$$

This concludes the proof.

Theorem 3.5. Let $T$ a set of indices, $V_{t}$ and $W_{t}$ finite dimensional Hilbert spaces, for every $t \in T$. Let $\boldsymbol{\mu} \in S Y^{2}\left(D ;\left(V_{t}\right)_{t \in T}\right)$ and $\boldsymbol{\nu} \in S Y^{2}\left(D ;\left(V_{t} \times W_{t}\right)_{t \in T}\right)$. Assume that

$$
\tilde{\pi}_{V_{t}}\left(\boldsymbol{\nu}_{t}\right)=\boldsymbol{\mu}_{t}
$$

for every $t \in T$. Then there exist a $\left(D, \mathcal{L}^{d}\right)$-probability space $(D \times \Omega, \mathcal{B}(D) \otimes \mathcal{F}, P)$ and a stochastic process $\left(\boldsymbol{Z}_{t}, \boldsymbol{Y}_{t}\right)_{t \in T}$ with

$$
\begin{gathered}
Z_{t} \in \mathrm{L}^{2}\left(D \times \Omega ; V_{t}\right), \\
\boldsymbol{Y}_{t} \in \mathrm{L}^{2}\left(D \times \Omega ; W_{t}\right),
\end{gathered}
$$

for every $t \in T$, such that

$$
\left(\pi_{D},\left(\boldsymbol{Z}_{t}\right)_{t \in F}\right)(P)=\boldsymbol{\mu}_{F},
$$

for every nonempty finite subset $F$ of $T$, and

$$
\left(\pi_{D}, \boldsymbol{Z}_{t}, \boldsymbol{Y}_{t}\right)(P)=\boldsymbol{\nu}_{t}
$$

for every $t \in T$.

Proof. We want to construct from $\boldsymbol{\mu}$ and $\boldsymbol{\nu}$ a unique compatible system and to apply to it Theorem 3.3.

Fix a nonempty finite subset $F$ of $T$. Denote by $\left(\boldsymbol{\mu}_{F}^{x}\right)_{x \in D},\left(\boldsymbol{\nu}_{t}^{x}\right)_{x \in D}$ the disintegrations with respect to $\mathcal{L}^{d}$ of $\boldsymbol{\mu}_{F}$, and $\boldsymbol{\nu}_{t}, t \in F$, respectively. As observed in (3.12), $\pi_{V_{t}}\left(\boldsymbol{\nu}_{t}^{x}\right)=\boldsymbol{\mu}_{t}^{x}$, for a.e. $x \in D$, for every $t \in F$. Hence, for a.e. $x \in D$, we can write

$$
\boldsymbol{\nu}_{t}^{x}=\int_{V_{t}}\left(\boldsymbol{\nu}_{t}^{x}\right)^{v_{t}} \mathrm{~d} \boldsymbol{\mu}_{t}^{x}\left(v_{t}\right) .
$$

Using the fact that the disintegration is a measurable family, a Dynkin class argument, and Lemma 3.4, we can deduce that

$$
\left(x,\left(v_{t}\right)_{t \in F}\right) \mapsto\left(\underset{t \in F}{\otimes}\left(\boldsymbol{\nu}_{t}^{x}\right)^{v_{t}}\right)(B)
$$

is a Borel measurable function, for every Borel subset $B$ of $\prod_{t \in F} W_{t}$. In particular for a.e. $x \in D$ the function $\left(v_{t}\right)_{t \in F} \mapsto\left(\otimes_{t \in F}\left(\boldsymbol{\nu}_{t}^{x}\right)^{v_{t}}\right)(B)$ is Borel measurable; hence, for every Borel sets $A \subseteq \prod_{t \in F} V_{t}$ and $B \subseteq \prod_{t \in F} W_{t}$, 
we can define a measure $\tilde{\boldsymbol{\nu}}_{F}^{x}$ on $\prod_{t \in F}\left(V_{t} \times W_{t}\right)$ by

$$
\tilde{\boldsymbol{\nu}}_{F}^{x}(A \times B):=\int_{A}\left(\underset{t \in F}{\otimes}\left(\boldsymbol{\nu}_{t}^{x}\right)^{v_{t}}\right)(B) \mathrm{d} \boldsymbol{\mu}_{F}^{x}\left(\left(v_{t}\right)_{t \in F}\right),
$$

for a.e. $x \in D$.

By construction, for a.e. $x \in D, \tilde{\boldsymbol{\nu}}_{F}^{x}$ is a probability measure with the properties

$$
\begin{aligned}
& \pi_{t \in F} V_{t}\left(\tilde{\boldsymbol{\nu}}_{F}^{x}\right)=\boldsymbol{\mu}_{F}^{x}, \\
& \pi_{V_{t} \times W_{t}}\left(\tilde{\boldsymbol{\nu}}_{F}^{x}\right)=\boldsymbol{\nu}_{t}^{x},
\end{aligned}
$$

for every $t \in F$.

The Borel measurability of the function in (3.15) guarantees that $\left(\tilde{\boldsymbol{\nu}}_{F}^{x}\right)_{x \in D}$ is a measurable family of probability measures on $\prod_{t \in F}\left(V_{t} \times W_{t}\right)$ and we can define

$$
\tilde{\boldsymbol{\nu}}_{F}:=\int_{D} \tilde{\boldsymbol{\nu}}_{F}^{x} \mathrm{~d} x
$$

It is easy to check that, for $F$ running over all nonempty finite subsets of $T, \tilde{\boldsymbol{\nu}}_{F} \in Y^{2}\left(D ; \prod_{t \in F}\left(V_{t} \times W_{t}\right)\right)$ and satisfies the compatibility condition; hence we have $\tilde{\boldsymbol{\nu}} \in S Y^{2}\left(D ;\left(V_{t} \times W_{t}\right)_{t \in T}\right)$. Moreover, thanks to (3.17) and (3.18),

$$
\tilde{\pi}_{\prod_{t \in F} V_{t}}\left(\tilde{\boldsymbol{\nu}}_{F}\right)=\boldsymbol{\mu}_{F}
$$

for every nonempty finite subset $F$ of $T$ and

$$
\tilde{\pi}_{V_{t} \times W_{t}}\left(\tilde{\boldsymbol{\nu}}_{t}\right)=\boldsymbol{\nu}_{t}
$$

for every $t \in T$.

Applying Theorem 3.3 to $\tilde{\boldsymbol{\nu}}$, we obtain a $\left(D, \mathcal{L}^{d}\right)$-probability space $(D \times \Omega, \mathcal{B}(D) \otimes \mathcal{F}, P)$ and a stochastic process $\left(\boldsymbol{Z}_{t}, \boldsymbol{Y}_{t}\right)_{t \in T}$, with $\left(\boldsymbol{Z}_{t}, \boldsymbol{Y}_{t}\right): D \times \Omega \rightarrow V_{t} \times W_{t}$, such that

$$
\left(\pi_{D},\left(\boldsymbol{Y}_{t}, \boldsymbol{Z}_{t}\right)_{t \in F}\right)(P)=\tilde{\boldsymbol{\nu}}_{F},
$$

for every nonempty finite subset $F$ of $T$. Using the construction of $\left(\boldsymbol{Z}_{t}, \boldsymbol{Y}_{t}\right)$ and (3.19), (3.20), we can obtain the thesis.

If the time set is an interval $[0, T] \subset \mathbb{R}$ and $\Xi_{t} \equiv \Xi$ for every $t \in[0, T]$, the notion of variation of a stochastic process $\left(\boldsymbol{Y}_{t}\right)_{t \in[0, T]}$ on a $\left(D, \mathcal{L}^{d}\right)$-probability space, with $\boldsymbol{Y}_{t} \in \mathrm{L}^{1}(D \times \Omega ; \Xi)$, is defined in the usual way: for every time interval $[a, b] \subseteq[0, T]$ we set

$$
\begin{aligned}
\operatorname{Var}(\boldsymbol{Y}, P ; a, b) & :=\sup \sum_{i=1}^{k} \int_{D \times \Omega}\left|\boldsymbol{Y}_{t_{i}}(x, \omega)-\boldsymbol{Y}_{t_{i-1}}(x, \omega)\right| \mathrm{d} P(x, \omega) \\
& =\sup \sum_{i=1}^{k}\left\|\boldsymbol{Y}_{t_{i}}-\boldsymbol{Y}_{t_{i-1}}\right\|_{1},
\end{aligned}
$$

where the supremum is taken over all finite partitions $a=t^{0}<\ldots<t^{k}=b$ of the interval $[a, b]$ (with the convention $\operatorname{Var}(\boldsymbol{Y}, P ; a, b)=0$, if $a=b)$. 
Using the correspondence between stochastic processes and compatible systems we define the variation of $\boldsymbol{\mu} \in S Y^{2}([0, T], D ; \Xi)$ on $[a, b] \subseteq[0, T]$ by

$$
\operatorname{Var}(\boldsymbol{\mu} ; a, b):=\sup \sum_{i=1}^{k} \int_{D \times \Xi^{k+1}}\left|\xi_{i}-\xi_{i-1}\right| \mathrm{d} \boldsymbol{\mu}_{t_{0} \ldots t_{k}}\left(x, \xi_{0}, \ldots, \xi_{k}\right)
$$

where the supremum is taken over all finite partitions $a=t_{0}<\ldots<t_{k}=b$ of the interval $[a, b]$ (with the convention $\operatorname{Var}(\boldsymbol{\mu} ; a, b)=0$, if $a=b)$.

From these definitions, if $\boldsymbol{\mu}$ is the compatible system associated to the stochastic process $\boldsymbol{Y}$ we have $\operatorname{Var}(\boldsymbol{Y}, P ; a, b)=\operatorname{Var}(\boldsymbol{\mu} ; a, b)$.

\section{Some technical tools}

For technical reasons it is more convenient to deal with Young measures and compatible systems than with random variables and stochastic processes; therefore we want to point out some results about measures which will be needed in the sequel.

We say that a sequence $\mu_{k}$ in $Y(D ; \Xi)$ weakly* converges if it converges in the weak* topology of $M_{b}(D \times \Xi)$.

Remark 4.1. Since the total variation of a Young measure $\mu$ is $|\mu|(D \times \Xi)=\mathcal{L}^{d}(D)=1, Y(D ; \Xi)$ is contained in a bounded subset of the dual of a separable Banach space, therefore it is metrizable with respect to the weak* topology.

We will say that $\mu_{k} \rightarrow \mu 2$-weakly* if the second moments of $\mu_{k}$ are equibounded and $\mu_{k} \rightarrow \mu$ weakly*.

We recall that $Y(D ; \Xi)$ is not closed with respect to the weak* convergence, differently from $Y^{2}(D ; \Xi)$, which is closed under 2 -weakly* convergence, as can be deduced from the following remarks.

Remark 4.2. If $\mu_{k}$ is a sequence in $Y(D ; \Xi)$ and $\mu_{k}$ weakly* converges to some $\mu \in M_{b}(D \times \Xi)$, then for every positive Carathéodory function $f$ on $D \times \Xi$ we have

$$
\int_{D \times \Xi} f(x, \xi) \mathrm{d} \mu(x, \xi) \leq \liminf _{k \rightarrow \infty} \int_{D \times \Xi} f(x, \xi) \mathrm{d} \mu_{k}(x, \xi),
$$

(see [18], Th. 4). Moreover, for every bounded Carathéodory function $f$ with compact support on $D \times \Xi$, we have

$$
\int_{D \times \Xi} f(x, \xi) \mathrm{d} \mu_{k}(x, \xi) \rightarrow \int_{D \times \Xi} f(x, \xi) \mathrm{d} \mu(x, \xi) .
$$

Therefore, if a sequence $\left(\mu_{k}\right)_{k} \subset Y^{2}(D ; \Xi) 2$-weakly* converges to a measure $\mu \in M_{b}(D \times \Xi)$, then $\mu \in$ $Y^{2}(D ; \Xi)$; moreover from the previous remark we can deduce that a sequence in $Y^{2}(D ; \Xi)$ with equibounded second moments has always a subsequence which converges 2 -weakly*.

The following remark is a slight modification of [17], Proposition 6.5.

Remark 4.3. If $\mu_{k} \rightarrow \mu 2$-weakly*, for every Carathéodory function $f: D \times \Xi \rightarrow \mathbb{R}$, with $|f(x, \xi)| \leq$ $a(x)+b(x)|\xi|$, for every $x \in D, \xi \in \Xi$, for suitable $b \in \mathrm{L}^{2}(D)$ and $a \in \mathrm{L}^{1}(D)$, it holds

$$
\int_{D \times \Xi} f(x, \xi) \mathrm{d} \mu_{k}(x, \xi) \longrightarrow \int_{D \times \Xi} f(x, \xi) \mathrm{d} \mu(x, \xi) .
$$

This allows to prove that if $\mu_{k} \rightarrow \mu 2$-weakly* $\mu_{k}, \mu \in Y^{2}\left(D ; \Xi_{1} \times \Xi_{2}\right)$, then $\tilde{\pi}_{i}\left(\mu_{k}\right) \rightarrow \tilde{\pi}_{i}(\mu)$ in $Y^{2}\left(D ; \Xi_{i}\right)$, as $k \rightarrow \infty$, for $i=1,2$.

Moreover we have the following result, where for every $g \in \mathrm{L}^{2}(X ; \Xi)$ the translation map $\mathcal{T}_{g}$ from $D \times \Xi$ into itself is defined by $\mathcal{T}_{g}(x, \xi):=(x, \xi+g(x))$. 
Lemma 4.4. Let $\left(\mu_{k}\right)_{k}$ be a sequence in $Y^{2}(D ; \Xi)$, such that $\mu_{k} \rightarrow \mu 2$-weakly*. Then $\mathcal{T}_{g}\left(\mu_{k}\right) \rightarrow \mathcal{T}_{g}(\mu)$ 2 -weakly*, for every $g \in \mathrm{L}^{2}(X ; \Xi)$.

More in general:

Lemma 4.5. Let $\mu_{k} \rightarrow \mu 2$-weakly* and $g_{k}$ be a bounded sequence in $\mathrm{L}^{2}(D ; \Xi)$ such that $g_{k} \rightarrow g$ strongly in $\mathrm{L}^{1}(D ; \Xi)$. Then $\mathcal{T}_{g_{k}}\left(\mu_{k}\right) \rightarrow \mathcal{T}_{g}(\mu) 2$-weakly*.

Proof. Since $\left(g_{k}\right)_{k}$ is bounded in $\mathrm{L}^{2}(D ; \Xi)$, the second moments of $\mathcal{T}_{g_{k}}\left(\mu_{k}\right)$ are equibounded. We now prove that $\mathcal{T}_{g_{k}}\left(\mu_{k}\right) \rightarrow \mathcal{T}_{g}(\mu)$ weakly*. Since $\mathrm{C}_{0}(\mathrm{D} \times \Xi)$ is the closure of $\mathrm{C}_{0}^{\infty}(D \times \Xi)$ with respect to the norm $\|\cdot\|_{\infty}$, thanks to $(2.2)$, to prove that $\mathcal{T}_{g_{k}}\left(\mu_{k}\right) \rightarrow \mathcal{T}_{g}(\mu)$ weakly* it is enough to show that

$$
\int_{D \times \Xi} f\left(x, \xi+g_{k}(x)\right) \mathrm{d} \mu_{k}(x, \xi) \rightarrow \int_{D \times \Xi} f(x, \xi+g(x)) \mathrm{d} \mu(x, \xi),
$$

for every $f \in \mathrm{C}_{0}^{\infty}(D \times \Xi)$.

Let $f \in \mathrm{C}_{0}^{\infty}(D \times \Xi)$, we have

$$
\begin{gathered}
\left|\int_{D \times \Xi} f(x, \xi+g(x)) \mathrm{d} \mu(x, \xi)-\int_{D \times \Xi} f\left(x, \xi+g_{k}(x)\right) \mathrm{d} \mu_{k}(x, \xi)\right| \leq \\
\left|\int_{D \times \Xi} f(x, \xi+g(x)) \mathrm{d} \mu(x, \xi)-\int_{D \times \Xi} f(x, \xi+g(x)) \mathrm{d} \mu_{k}(x, \xi)\right| \\
\quad+\int_{D \times \Xi}\left|f(x, \xi+g(x))-f\left(x, \xi+g_{k}(x)\right)\right| \mathrm{d} \mu_{k}(x, \xi) .
\end{gathered}
$$

By the Lipschitz continuity of $f$ and (2.2), the last line can be estimated by $c\left\|g-g_{k}\right\|_{1}$ for a positive constant $c$; Lemma 4.4 implies now the thesis.

If we deal with Young measures generated by gradients, the following lemma, in the version of Fonseca et al. [7], Lemma 1.2, can be very useful.

Lemma 4.6 (decomposition lemma). Let $\left(v_{j}\right)_{j}$ be a bounded sequence in $\mathrm{H}^{1}(D ; \Xi)$. Then there exists a subsequence $\left(v_{j_{k}}\right)_{k}$ of $\left(v_{j}\right)_{j}$, and another sequence $\left(w_{k}\right)_{k}$ bounded in $\mathrm{H}^{1}(D ; \Xi)$, such that

$$
\mathcal{L}^{d}\left(\left\{v_{j_{k}} \neq w_{k} \text { or } \nabla v_{j_{k}} \neq \nabla w_{k}\right\}\right) \rightarrow 0,
$$

as $k \rightarrow \infty$, and $\left(\left|\nabla w_{k}\right|^{2}\right)_{k}$ is equiintegrable.

Note that condition (4.2) implies that both sequences $\left(\nabla v_{j_{k}}\right)_{k}$ and $\left(\nabla w_{k}\right)_{k}$ generate the same Young measure, i.e., $\delta_{\nabla v_{j_{k}}}$ and $\delta_{\nabla w_{k}}$ converge to the same Young measure.

Using part of the arguments of [7] and a more careful diagonalization argument, it can be proved the following lemma.

Lemma 4.7. Let $\left(v_{j}\right)_{j}$ be a bounded sequence in $\mathrm{L}^{2}(D ; \Xi)$ such that there exists a Young measure $\mu \in Y^{2}(D ; \Xi)$ with $\delta_{v_{j}} \rightarrow \mu$ weakly*. Then there exists another sequence $\left(w_{j}\right)_{j}$, bounded in $\mathrm{L}^{2}(D ; \Xi)$, such that

$$
\mathcal{L}^{d}\left(\left\{v_{j} \neq w_{j}\right\}\right) \rightarrow 0
$$

as $j \rightarrow \infty$, and $\left(\left|w_{j}\right|^{2}\right)_{j}$ is equiintegrable.

The following theorem (see [2]) gives an important convergence result in case we deal with equiintegrable sequences. 
Theorem 4.8 (fundamental theorem for Young measures). Given a Young measure $\mu$, generated by a sequence of functions $\left(u_{j}\right)_{j}$, and a function $f \in \mathrm{C}(\Xi ; \mathbb{R})$ such that the sequence $\left(f\left(u_{j}\right)\right)_{j}$ is weakly sequentially relatively compact in $\mathrm{L}^{1}(D)$, then

$$
f\left(u_{j}\right) \rightarrow f_{\mu} \text { weakly in } \mathrm{L}^{1}(D)
$$

where the function $f_{\mu} \in \mathrm{L}^{1}(D)$ is defined by $f_{\mu}(x):=\int_{\Xi} f(\xi) \mathrm{d} \mu^{x}(\xi)$ for a.e. $x \in D$. In particular

$$
\int_{D} f\left(u_{j}(x)\right) \mathrm{d} x \rightarrow \int_{D \times \Xi} f(\xi) \mathrm{d} \mu(x, \xi) .
$$

The barycentre of a Young measure $\mu \in Y^{2}(D ; \Xi)$ is the function $\operatorname{bar}(\mu) \in \mathrm{L}^{2}(D ; \Xi)$ defined as

$$
\operatorname{bar}(\mu)(x):=\int_{\Xi} \xi \mathrm{d} \mu^{x}(\xi),
$$

for a.e. $x \in D$.

Lemma 4.9. Let $\mu_{k}$ be a sequence in $Y^{2}(D ; \Xi)$, such that $\mu_{k} \rightarrow \mu 2$-weakly*. Assume that there exists a sequence of functions $v_{k} \in \mathrm{H}_{0}^{1}(D ; \Xi)$ such that $\nabla v_{k}=\operatorname{bar}\left(\mu_{k}\right)$ for every $k$. Then there exists a function $v \in \mathrm{H}_{0}^{1}(D ; \Xi)$ such that $v_{k} \rightarrow v$ weakly in $\mathrm{H}^{1}$ and $\nabla v=\operatorname{bar}(\mu)$.

Proof. Since $\left\|\nabla v_{k}\right\|_{2}^{2} \leq \int_{D \times \Xi}|\xi|^{2} \mathrm{~d} \mu_{k}(x, \xi)$ which is bounded uniformly with respect to $k$ by hypothesis, using Poincaré inequality we can deduce that there exists a subsequence $v_{k_{h}}$ and a function $v \in \mathrm{H}_{0}^{1}(D ; \Xi)$, such that $v_{k_{h}} \rightarrow v$ weakly in $\mathrm{H}^{1}$. Using the definition of barycentre and (4.3), we deduce that $\nabla v=\operatorname{bar}(\mu)$ and hence $\nabla v_{k_{h}} \rightarrow \operatorname{bar}(\mu)$ weakly in $\mathrm{L}^{2}(D ; \Xi)$; together with Poincaré inequality, this implies that the whole sequence $v_{k}$ converges to $v$ weakly in $\mathrm{H}^{1}$.

The space $S Y^{2}\left(D ; \prod_{t \in T} \Xi_{t}\right)$ will be equipped with the weakest topology for which the maps $\boldsymbol{\mu} \mapsto \boldsymbol{\mu}_{F}$ from $S Y^{2}\left(D ; \prod_{t \in T} \Xi_{t}\right)$ into $Y^{2}\left(D ; \prod_{t \in F} \Xi_{t}\right)$, endowed with the weak ${ }^{*}$ topology of $M_{b}\left(D \times \prod_{t \in F} \Xi_{t}\right)$, are continuous for every nonempty finite subset $F$ of $T$. We will refer to this topology as the weak* topology of $S Y^{2}\left(D ; \prod_{t \in T} \Xi_{t}\right)$.

From now on we consider the case in which the time set is an interval $[0, T]$. A compatible system of Young measures with finite second moments $\boldsymbol{\mu}$ is said to be left continuous if for every finite sequence $t_{1}, \ldots, t_{m}$ in $[0, T]$, with $t_{1}<\ldots<t_{m}$, the following property holds:

$$
\boldsymbol{\mu}_{s_{1} \ldots s_{m}} \rightarrow \boldsymbol{\mu}_{t_{1} \ldots t_{m}} \text { weakly* in } Y^{2}\left(D ; \Xi^{m}\right)
$$

as $s_{i} \rightarrow t_{i}$, with $s_{i} \in[0, T]$ and $s_{i} \leq t_{i}$. We will denote the space of all left continuous compatible systems by $S Y_{-}^{2}([0, T], D ; \Xi)$.

The following theorem can be considered as a version of Helly's Theorem.

Theorem 4.10. Let $\boldsymbol{\mu}^{k}$ be a sequence in $S Y^{2}([0, T], D ; \Xi)$ such that

$$
\begin{gathered}
\sup _{k} \operatorname{Var}\left(\boldsymbol{\mu}^{k} ; 0, T\right) \leq C, \\
\sup _{t \in[0, T]} \sup _{k} \int_{D \times \Xi}|\xi|^{2} \mathrm{~d} \boldsymbol{\mu}_{t}^{k}(x, \xi) \leq C^{*},
\end{gathered}
$$

for finite constants $C$ and $C^{*}$. Then there exist a subsequence, still denoted by $\boldsymbol{\mu}^{k}$, a set $\Theta \subset[0, T]$, containing 0 and such that $[0, T] \backslash \Theta$ is at most countable, and $\boldsymbol{\mu} \in S Y_{-}^{2}([0, T], D ; \Xi)$ with

$$
\begin{gathered}
\operatorname{Var}(\boldsymbol{\mu} ; 0, T) \leq C, \\
\int_{D \times \Xi}|\xi|^{2} \mathrm{~d} \boldsymbol{\mu}_{t}(x, \xi) \leq C^{*} \quad \text { for every } t \in[0, T],
\end{gathered}
$$


such that, for every nonempty finite subset $F$ of $\Theta$, we have

$$
\boldsymbol{\mu}_{F}^{k} \rightarrow \boldsymbol{\mu}_{F} 2 \text {-weakly*. }
$$

The proof of this theorem follows easily from Theorem 8.10 of [5], since every Young measure can be seen as a generalized Young measure and our more restrictive hypotheses force the limit to be an element of $S Y^{2}([0, T], D ; \Xi)$.

If $H: \Xi \rightarrow[0,+\infty)$ is positively homogeneous of degree one and satisfies the triangle inequality, we can define the $H$-variation of $\boldsymbol{\mu} \in S Y^{2}([0, T], D ; \Xi)$ on the time interval $[a, b] \subseteq[0, T]$ as

$$
\operatorname{Var}_{H}(\boldsymbol{\mu} ; a, b):=\sup \sum_{i=1}^{k} \int_{D \times \Xi^{k+1}} H\left(\xi_{i}-\xi_{i-1}\right) \mathrm{d} \boldsymbol{\mu}_{t_{0} \ldots t_{k}}\left(x, \xi_{0}, \ldots, \xi_{k}\right),
$$

where the supremum is taken over all finite partitions $a=t_{0}<\ldots<t_{k}=b$ of the interval $[a, b]$ (with the convention $\operatorname{Var}_{H}(\boldsymbol{\mu} ; a, b)=0$, if $\left.a=b\right)$.

Adapting the argument in [5] (Th. 8.11), it can be proved the following lemma.

Lemma 4.11. Let $\boldsymbol{\mu}^{k}$ be a sequence in $S Y^{2}([0, T], D ; \Xi)$. Suppose that there exist a dense set $\Theta \subset[0, T]$ containing 0 and $\boldsymbol{\mu} \in S Y_{-}^{2}([0, T], D ; \Xi)$, such that

$$
\boldsymbol{\mu}_{F}^{k} \rightarrow \boldsymbol{\mu}_{F} \quad 2-w e a k l y^{*}
$$

for every nonempty finite subset $F$ in $\Theta$; then

$$
\operatorname{Var}_{H}(\boldsymbol{\mu} ; 0, T) \leq \liminf _{k \rightarrow \infty} \operatorname{Var}_{H}\left(\boldsymbol{\mu}^{k} ; 0, T\right)
$$

for every positively one homogeneous function $H: \Xi \rightarrow[0,+\infty)$ satisfying the triangle inequality.

Definition 4.12. Fix a finite sequence $0=t_{1}<\ldots<t_{m}=T$ in $[0, T]$. For every $\mu \in Y^{2}\left(D ; \Xi^{m}\right)$, it is possible to define the piecewise constant interpolation $\boldsymbol{\mu}^{p w c} \in S Y^{2}([0, T], D ; \Xi)$ in the following way. For every finite sequence $\tau_{1}<\ldots<\tau_{n}$ of elements of $[0, T]$ let $\rho_{\tau_{1} \ldots \tau_{n}}: D \times \Xi^{m} \times \mathbb{R} \rightarrow D \times \Xi^{n} \times \mathbb{R}$ be defined by

$$
\rho_{\tau_{1} \ldots \tau_{n}}\left(x, \xi_{t_{1}}, \ldots, \xi_{t_{m}}\right):=\left(x, \xi_{\tau_{1}}, \ldots, \xi_{\tau_{n}}\right),
$$

with $\xi_{\tau_{i}}=\xi_{t_{j}}$, where $j$ is the largest index such that $t_{j} \leq \tau_{i}$. The compatible system of Young measures with finite second moments $\boldsymbol{\mu}^{p w c}$ is then defined by

$$
\boldsymbol{\mu}_{\tau_{1} \ldots \tau_{n}}^{p w c}:=\rho_{\tau_{1} \ldots \tau_{n}}(\mu) .
$$

Lemma 4.13. Let $\left(\boldsymbol{\mu}^{n}\right)_{n}$ and $\left(\boldsymbol{\nu}^{n}\right)_{n}$ be sequences in $S Y^{2}\left([0, T], D ; \Xi_{1}\right)$ and $S Y^{2}\left([0, T], D ; \Xi_{1} \times \Xi_{2}\right)$, respectively, satisfying $\tilde{\pi}_{\Xi_{1}}\left(\boldsymbol{\nu}_{t}^{n}\right)=\boldsymbol{\mu}_{t}^{n}$, for every $t \in[0, T]$. Assume that

$$
\sup _{t \in[0, T]} \sup _{n} \int_{D \times \Xi_{1} \times \Xi_{2}}\left|\left(\xi_{1}, \xi_{2}\right)\right|^{2} \mathrm{~d} \boldsymbol{\nu}_{t}^{n}\left(x, \xi_{1}, \xi_{2}\right) \leq C,
$$

for a positive constant $C$, and that there exist a subset $\Theta$ of $[0, T]$, containing 0 , with $\mathcal{L}^{1}([0, T] \backslash \Theta)=0$, and $\boldsymbol{\mu} \in S Y_{-}^{2}\left([0, T], D ; \Xi_{1}\right)$ such that

$$
\boldsymbol{\mu}_{t_{1} \ldots t_{m}}^{n} \rightarrow \boldsymbol{\mu}_{t_{1} \ldots t_{m}}, \quad 2 \text {-weakly*, }
$$

for every $t_{1}<\ldots<t_{m}$ in $\Theta$. For every $t \in \Theta$ let $\left(n_{k}^{t}\right)_{k}$ be an increasing sequence of integers; then there exists $\boldsymbol{\nu} \in S Y^{2}\left([0, T], D ; \Xi_{1} \times \Xi_{2}\right)$, such that $\tilde{\pi}_{\Xi_{1}}\left(\boldsymbol{\nu}_{t}\right)=\boldsymbol{\mu}_{t}$, for every $t \in[0, T]$ and satisfying the following properties: 
(1) for every $t \in \Theta$, there exists a subsequence $\boldsymbol{\nu}_{t}^{n_{k, i}^{t}}$ of $\boldsymbol{\nu}_{t}^{n_{k}^{t}}$ such that

$$
\boldsymbol{\nu}_{t}^{n_{k, i}^{t}} \rightarrow \boldsymbol{\nu}_{t} \quad 2 \text {-weakly*; }
$$

(2) for every $t \in[0, T] \backslash \Theta$, there exists a sequence $s^{j}$ in $\Theta$, converging to $t$, with $s^{j} \leq t$, such that

$$
\boldsymbol{\nu}_{s^{j}} \rightarrow \boldsymbol{\nu}_{t} 2 \text {-weakly* }
$$

The proof of this lemma was contained in a preliminary version of [6].

Proof. Fix $t \in \Theta$; thanks to (4.10), we can deduce that there exist $\nu_{t}^{0} \in Y^{2}\left(D ; \Xi_{1} \times \Xi_{2}\right)$ and a subsequence $\left(\boldsymbol{\nu}_{t}^{n_{k, i}^{t}}\right)_{i}$ of $\left(\boldsymbol{\nu}_{t}^{n_{k}^{t}}\right)_{k}$, satisfying (4.11). Thanks to Remarks 4.2 and 4.3, for every $t \in \Theta$ we have

$$
\int_{D \times \Xi_{1} \times \Xi_{2}}\left|\left(\xi_{1}, \xi_{2}\right)\right|^{2} \mathrm{~d} \nu_{t}^{0}\left(x, \xi_{1}, \xi_{2}\right) \leq C
$$

and $\tilde{\pi}_{\Xi_{1}}\left(\nu_{t}^{0}\right)=\boldsymbol{\mu}_{t}$.

Consider now the sets $B_{t}$ defined in the following way:

- if $t \in \Theta, B_{t}$ denotes the collection of all $\nu \in S Y^{2}\left([0, T], D ; \Xi_{1} \times \Xi_{2}\right)$ such that the second moments of $\boldsymbol{\nu}_{s}$ are bounded by the constant $C$ appearing in (4.10), for every $s \in[0, T]$, and satisfying $\boldsymbol{\nu}_{t}=\nu_{t}^{0}$;

- if $t \notin \Theta, B_{t}$ is the collection of all $\boldsymbol{\nu} \in S Y^{2}\left([0, T], D ; \Xi_{1} \times \Xi_{2}\right)$, such that the second moments of $\boldsymbol{\nu}_{s}$ are bounded by the constant $C$ appearing in (4.10), for every $s \in[0, T]$, and for which there exists a sequence $s^{j}$ in $\Theta$, converging to $t$ with $s^{j} \leq t$, such that $\nu_{s^{j}}^{0} \rightarrow \boldsymbol{\nu}_{t}$, weakly*.

For every $t \in[0, T], B_{t} \neq \varnothing$ : indeed, if $t \in \Theta$ it comes immediately from Remark 3.2 applied to $\left(\boldsymbol{\nu}_{s}\right)_{s \in[0, T]} \in$ $Y^{2}(D ; \Xi)^{[0, T]}$ defined by $\nu_{s}:=\nu_{t}^{0}$ for every $s \in[0, T]$; if $t \notin \Theta$, thanks to (4.13), there exist $s^{j}$, with $s^{j} \in \Theta$, $s^{j} \rightarrow t$ and $s^{j} \leq t$, and $\mu \in Y^{2}\left(D ; \Xi_{1} \times \Xi_{2}\right)$, such that $\nu_{s^{j}}^{0} \rightarrow \mu 2$-weakly*, hence the second moment of $\mu$ is bounded by $C$ and we can apply Remark 3.2 to $\left(\boldsymbol{\nu}_{s}\right)_{s \in[0, T]} \in Y^{2}(D ; \Xi)^{[0, T]}$ defined by $\boldsymbol{\nu}_{s}:=\mu$, for every $s \in[0, T]$, and find an element of $B_{t}$. Using Remark 4.1 we can see that the set of all Young measures $\mu$ for which there exists a sequence $s^{j} \rightarrow t$ with $s^{j} \leq t$ and $\nu_{s^{j}}^{0} \rightarrow \mu$ weakly* is sequentially closed with respect to the weak* $^{*}$ topology (thanks to (4.13)); moreover we observe that the set of all $\boldsymbol{\nu} \in S Y^{2}([0, T], D ; \Xi)$ with the second moments equibounded by the constant $C$ is closed in the weak topology of $S Y^{2}([0, T], D ; \Xi)$, therefore, using again Remark 4.1, we can conclude that, for every $t \in[0, T], B_{t}$ are closed subsets of $S Y^{2}\left([0, T], D ; \Xi_{1} \times \Xi_{2}\right)$, endowed with the weak* topology. Moreover the family has the finite intersection property (for every finite sequence $t_{1}<\ldots<t_{m}$ in $[0, T]$, using Definition 4.12 , we can find an element belonging to $B_{t_{1}} \cap \ldots \cap B_{t_{m}}$ ) and is contained in the set of all $\boldsymbol{\nu} \in S Y^{2}\left([0, T], D ; \Xi_{1} \times \Xi_{2}\right)$ for which the second moments of $\boldsymbol{\nu}_{t}$ are uniformly bounded by the constant $C$ appearing in (4.10); since, thanks to Tychonoff's Theorem, this is a compact subset of $S Y^{2}\left([0, T], D ; \Xi_{1} \times \Xi_{2}\right)$, endowed with the weak* topology, we can conclude that there exists $\boldsymbol{\nu}$ belonging to $B_{t}$, for every $t \in[0, T]$. By construction $\boldsymbol{\nu}$ satisfies (1) and (2) and from the left continuity of $\boldsymbol{\mu}$ we can deduce that $\tilde{\pi}_{\Xi_{1}}\left(\boldsymbol{\nu}_{t}\right)=\boldsymbol{\mu}_{t}$, for every $t \in[0, T]$, as required.

\section{MeChaniCAL MODEL}

The reference configuration $D$ is a bounded connected open subset of $\mathbb{R}^{d}$ with Lipschitz boundary $\partial D=$ $\Gamma_{0} \cup \Gamma_{1}$, where $\Gamma_{0}$ is assumed to be a nonempty closed subset of $\partial D$ with $\mathcal{H}^{d-1}\left(\Gamma_{0}\right) \neq 0$, and $\Gamma_{1}=\partial D \backslash \Gamma_{0}$. Without loss of generality, we also assume for simplicity that $\mathcal{L}^{d}(D)=1$.

We will indicate the deformation by $v$ and the internal variable by $z$. We will denote the stored energy density by $W: \mathbb{R}^{m} \times \mathbb{R}^{N \times d} \rightarrow[0,+\infty)$ and the dissipation rate density by $H: \mathbb{R}^{m} \rightarrow[0,+\infty)$. For every $\theta, \tilde{\theta} \in \mathbb{R}^{m}$ and $F \in \mathbb{R}^{N \times d}$, we will make the following assumptions: 
(W.1) there exist positive constants $c, C$ such that

$$
c\left(|\theta|^{2}+|F|^{2}\right)-C \leq W(\theta, F) \leq C\left(1+|\theta|^{2}+|F|^{2}\right)
$$

(W.2) $W(\theta, \cdot)$ is of class $\mathrm{C}^{1}$,

$$
\left|\frac{\partial W}{\partial F}(\theta, F)\right| \leq C(1+|\theta|+|F|)
$$

and

$$
|W(\theta+\tilde{\theta}, F)-W(\theta, F)| \leq C|\tilde{\theta}|(1+|\theta|+|\tilde{\theta}|+|F|)
$$

(H.1) $H$ is positively homogeneous of degree one and convex;

(H.2) there exists a positive constant $\lambda$, such that $\frac{1}{\lambda}|\theta| \leq H(\theta) \leq \lambda|\theta|$.

Let $\mathcal{W}$ be the functional $\mathcal{W}(z, v):=\int_{D} W(z(x), \nabla v(x)) \mathrm{d} x$, for every $z \in \mathrm{L}^{2}\left(D ; \mathbb{R}^{m}\right)$ and every $v \in$ $\mathrm{H}^{1}\left(D ; \mathbb{R}^{N}\right)$, and $\mathcal{H}$ the functional $\mathcal{H}(z):=\int_{D} H(z(x)) \mathrm{d} x$, for every $z \in \mathrm{L}^{1}\left(D ; \mathbb{R}^{m}\right)$.

Given two distinct times $s<t$, the global dissipation of a possibly discontinuous function $z:[0, T] \rightarrow$ $\mathrm{L}^{2}\left(D ; \mathbb{R}^{m}\right)$ in the interval $[s, t]$ will be

$$
\operatorname{Var}_{H}(\boldsymbol{z} ; s, t):=\sup \sum_{i=1}^{k} \mathcal{H}\left(\boldsymbol{z}\left(\tau_{i}\right)-\boldsymbol{z}\left(\tau_{i-1}\right)\right)
$$

where the supremum will be taken among all finite partitions $s=\tau_{0}<\tau_{1}<\ldots<\tau_{k}=t$.

The external load at time $t$ and the prescribed boundary datum on $\Gamma_{0}$ at time $t$ are denoted by $l(t)$ and $\boldsymbol{\varphi}(t)$, respectively; we assume $\boldsymbol{l} \in A C\left([0, T] ; \mathrm{H}^{1}\left(D ; \mathbb{R}^{N}\right)^{*}\right)$ and $\boldsymbol{\varphi} \in A C\left([0, T] ; \mathrm{H}^{1}\left(D ; \mathbb{R}^{N}\right)\right)$.

The kinematically admissible values at time $t$ for $z$ and $v$ are those which make the total energy finite and satisfy the boundary condition, i.e., $v=\varphi(t)$ on $\Gamma_{0} \mathcal{H}^{d-1}$-a.e. (in the sense of traces). From the previous assumption it follows that the kinematically admissible values at time $t$ are contained in $\mathrm{L}^{2}\left(D ; \mathbb{R}^{m}\right) \times \mathcal{A}(t)$, where $\mathcal{A}(t)=\mathrm{H}_{\Gamma_{0}}^{1}(\varphi(t)):=\left\{v \in \mathrm{H}^{1}\left(D ; \mathbb{R}^{N}\right): v=\varphi(t) \mathcal{H}^{d-1}\right.$-a.e. on $\left.\Gamma_{0}\right\}$.

\section{Globally stable quasistatic evolution for Young measures}

\subsection{Admissible set in terms of stochastic processes}

Now we describe the set of admissible stochastic processes in which we look for a solution of our quasistatic evolution problem: the definition takes into account approximation properties with functions which satisfy the boundary condition.

Definition 6.1. Given $A \subset \mathbb{R}$ and $\boldsymbol{w}: A \rightarrow \mathrm{H}^{1}\left(D ; \mathbb{R}^{N}\right)$, we define $A Y_{s p}(A, \boldsymbol{w})$ as the set of all stochastic processes $\left(\boldsymbol{Z}_{t}, \boldsymbol{Y}_{t}\right)_{t \in A}$ on a $\left(D, \mathcal{L}^{d}\right)$-probability space $(D \times \Omega, \mathcal{B}(D) \otimes \mathcal{F}, P)$ with

$$
\begin{gathered}
\boldsymbol{Z}_{t} \in \mathrm{L}^{2}\left(D \times \Omega ; \mathbb{R}^{m}\right), \\
\boldsymbol{Y}_{t} \in \mathrm{L}^{2}\left(D \times \Omega ; \mathbb{R}^{N \times d}\right),
\end{gathered}
$$

satisfying the following property: for every finite sequence $t_{1}<\ldots<t_{n}$ in $A$ there exist sequences $\left(z_{i}^{k}\right)_{k} \subset$ $\mathrm{L}^{2}\left(D ; \mathbb{R}^{m}\right),\left(v_{i}^{k}\right)_{k} \subset \mathrm{H}_{\Gamma_{0}}^{1}\left(\boldsymbol{w}\left(t_{i}\right)\right)$, for $i=1, \ldots, n$ such that 
$\left(\operatorname{ap}_{\mathrm{sp}} 1\right)$ we have

$$
\left(\pi_{D}, z_{1}^{k}, \ldots, z_{n}^{k}\right)(P) \rightarrow\left(\pi_{D}, Z_{t_{1}}, \ldots, Z_{t_{n}}\right)(P)
$$

2 -weakly* as $k \rightarrow \infty$;

$\left(\operatorname{ap}_{\mathrm{sp}} 2\right)$ for every $i=1, \ldots, n$, there exists a subsequence, possibly depending on $i,\left(z_{i}^{k_{j}^{i}}, v_{i}^{k_{j}^{i}}\right)_{j}$, such that

$$
\left(\pi_{D}, z^{k_{j}^{i}}, \nabla v^{k_{j}^{i}}\right)(P) \rightarrow\left(\pi_{D}, \boldsymbol{Z}_{t_{i}}, \boldsymbol{Y}_{t_{i}}\right)(P)
$$

2 -weakly* as $j \rightarrow \infty$.

\subsection{Admissible set in terms of Young measures}

The notion of admissible set is now presented in terms of Young measures.

Definition 6.2. Given $A \subset \mathbb{R}$ and $\boldsymbol{w}: A \rightarrow \mathrm{H}^{1}\left(D ; \mathbb{R}^{N}\right)$, we define $A Y(A, \boldsymbol{w})$ as the set of all pairs $(\boldsymbol{\nu}, \boldsymbol{\mu}) \in$ $Y^{2}\left(D ; \mathbb{R}^{m} \times \mathbb{R}^{N \times d}\right)^{A} \times S Y^{2}\left(A, D ; \mathbb{R}^{m}\right)$ satisfying the following property: for every finite sequence $t_{1}<\ldots<t_{n}$ in $A$ there exist sequences $\left(z_{i}^{k}\right)_{k} \subset \mathrm{L}^{2}\left(D ; \mathbb{R}^{m}\right),\left(v_{i}^{k}\right)_{k} \subset \mathrm{H}_{\Gamma_{0}}^{1}\left(\boldsymbol{w}\left(t_{i}\right)\right)$, for $i=1, \ldots, n$ such that

(ap1) we have

$$
\delta_{\left(z_{1}^{k}, \ldots, z_{n}^{k}\right)} \rightarrow \boldsymbol{\mu}_{t_{1} \ldots t_{n}}
$$

2 -weakly* as $k \rightarrow \infty$;

(ap2) for every $i=1, \ldots, n$, there exists a subsequence, possibly depending on $i,\left(z_{i}^{k_{j}^{i}}, v_{i}^{k_{j}^{i}}\right)_{j}$, such that

$$
\delta_{\left(z_{i}^{k_{j}^{i}}, \nabla v_{i}^{k_{j}^{i}}\right)} \rightarrow \boldsymbol{\nu}_{t_{i}}
$$

2 -weakly* as $j \rightarrow \infty$.

Remark 6.3. If $(\boldsymbol{\nu}, \boldsymbol{\mu}) \in A Y(A, \boldsymbol{w})$, then $\tilde{\pi}_{\mathbb{R}^{m}}\left(\boldsymbol{\nu}_{t}\right)=\boldsymbol{\mu}_{t}$, for every $t \in A$. Indeed, fixed $t \in A$, by definition there exist $\left(z^{k}\right)_{k} \subset \mathrm{L}^{2}\left(D ; \mathbb{R}^{m}\right),\left(v^{k}\right)_{k} \subset \mathrm{H}_{\Gamma_{0}}^{1}(\boldsymbol{w}(t))$ such that $\delta_{\left(z^{k}, \nabla v^{k}\right)} \rightarrow \boldsymbol{\nu}_{t} 2$-weakly* and $\delta_{z^{k}} \rightarrow \boldsymbol{\mu}_{t}$ 2-weakly*; in particular $\tilde{\pi}_{\mathbb{R}^{m}}\left(\delta_{\left(z^{k}, \nabla v^{k}\right)}\right) \rightarrow \tilde{\pi}_{\mathbb{R}^{m}}\left(\boldsymbol{\nu}_{t}\right)$ 2-weakly* and this prove the claim.

Remark 6.4. If $\left(\boldsymbol{Z}_{t}, \boldsymbol{Y}_{t}\right)_{t \in A} \in A Y_{s p}(A, \boldsymbol{w})$, we can define $(\boldsymbol{\nu}, \boldsymbol{\mu}) \in A Y(A, \boldsymbol{w})$ as

$$
\begin{gathered}
\boldsymbol{\nu}_{t}:=\left(\pi_{D}, \boldsymbol{Z}_{t}, \boldsymbol{Y}_{t}\right)(P) \text { for every } t \in A \\
\boldsymbol{\mu}_{t_{1} \ldots t_{n}}:=\left(\pi_{D}, \boldsymbol{Z}_{t_{1}}, \ldots, \boldsymbol{Z}_{t_{n}}\right)(P) \text { for every finite sequence } t_{1}<\ldots<t_{n} \text { in } A .
\end{gathered}
$$

On the other side, thanks to Remark 6.3 and Theorem 3.5, for every $(\boldsymbol{\nu}, \boldsymbol{\mu}) \in A Y(A, \boldsymbol{w})$ there exists a stochastic process $\left(\boldsymbol{Z}_{t}, \boldsymbol{Y}_{t}\right)_{t \in A} \in A Y_{s p}(A, \boldsymbol{w})$ such that, for every finite sequence $t_{1}<\ldots<t_{n}$ in $A$,

$$
\begin{gathered}
\left(\pi_{D}, \boldsymbol{Z}_{t_{1}}, \ldots, \boldsymbol{Z}_{t_{n}}\right)(P)=\boldsymbol{\mu}_{t_{1} \ldots t_{n}} \\
\left(\pi_{D}, \boldsymbol{Z}_{t_{i}}, \boldsymbol{Y}_{t_{i}}\right)(P)=\boldsymbol{\nu}_{t_{i}} \quad \text { for every } i=1, \ldots, n
\end{gathered}
$$

Remark 6.5. Thanks to decomposition Lemmas 4.6 and 4.7, given $(\boldsymbol{\nu}, \boldsymbol{\mu}) \in A Y(A, \boldsymbol{w})$ and a finite sequence $t_{1}<\ldots<t_{m}$ in $A$, we can always choose $z_{i}^{k} \in \mathrm{L}^{2}\left(D ; \mathbb{R}^{m}\right)$ and $v_{i}^{k} \in \mathrm{H}_{\Gamma_{0}}^{1}\left(\boldsymbol{w}\left(t^{i}\right)\right)$, for $i=1, \ldots, m$, in such a way that $\left|z_{i}^{k}\right|^{2}$ are equiintegrable, satisfy (6.1), and for every $i$ there exists a subsequence $\left(z_{i}^{k_{j}^{i}}, v_{i}^{k_{j}^{i}}\right)_{j}$ satisfying (6.2), 
such that $\left|\nabla v_{i}^{k_{j}^{i}}\right|^{2}$ are equiintegrable with respect to $j$. Hence, by Theorem 4.8, we can always assume that

$$
\begin{gathered}
\left\|\left(z_{1}^{k}, \ldots, z_{n}^{k}\right)\right\|_{2}^{2} \rightarrow \int_{D \times\left(\mathbb{R}^{m}\right)^{n}}\left|\left(\theta_{1}, \ldots, \theta_{n}\right)\right|^{2} \mathrm{~d} \boldsymbol{\mu}_{t_{1} \ldots t_{n}}\left(x, \theta_{1}, \ldots, \theta_{n}\right), \\
\left\|\left(z_{i}^{k_{j}^{i}}, \nabla v_{i}^{k_{j}^{i}}\right)\right\|_{2}^{2} \rightarrow \int_{D \times \mathbb{R}^{m} \times \mathbb{R}^{N \times d}}|(\theta, F)|^{2} \mathrm{~d} \boldsymbol{\nu}_{t_{i}}(x, \theta, \xi),
\end{gathered}
$$

as $j \rightarrow \infty$, for $i=1, \ldots, m$. This allows us to assume, without loss of generality, that

$$
\begin{gathered}
\sup _{k}\left\|z_{i}^{k}\right\|_{2}^{2} \leq C_{1}+1, \\
\sup _{j}\left\|\left(z_{i}^{k_{j}^{i}}, \nabla v_{i}^{k_{j}^{i}}\right)\right\|_{2}^{2} \leq C_{2}+1,
\end{gathered}
$$

with

$$
\begin{gathered}
C_{1}:=\sup _{i=1, \ldots, n} \int_{D \times \mathbb{R}^{m}}|\theta|^{2} \mathrm{~d} \boldsymbol{\mu}_{t_{i}}(x, \theta), \\
C_{2}:=\sup _{i=1, \ldots, n} \int_{D \times \mathbb{R}^{m} \times \mathbb{R}^{N \times d}}|(\theta, F)|^{2} \mathrm{~d} \boldsymbol{\nu}_{t_{i}}(x, \theta, F) .
\end{gathered}
$$

In the following two lemmas we want to point out some closure properties of $A Y(A, \boldsymbol{w})$.

Lemma 6.6. Let $(\boldsymbol{\nu}, \boldsymbol{\mu}) \in Y^{2}\left(D ; \mathbb{R}^{m} \times \mathbb{R}^{N \times d}\right)^{A} \times S Y^{2}\left(A, D ; \mathbb{R}^{m}\right)$, and assume that for every finite sequence $t_{1}<\ldots<t_{n}$ in $A$ there exists a sequence $\left(\boldsymbol{\nu}^{j}, \boldsymbol{\mu}^{j}\right)_{j}$ in $A Y\left(\left\{t_{1}, \ldots, t_{n}\right\}, \boldsymbol{w}\right)$, such that

$$
\boldsymbol{\mu}_{t_{1} \ldots t_{n}}^{j} \rightarrow \boldsymbol{\mu}_{t_{1} \ldots t_{n}} 2 \text {-weakly* }
$$

as $j \rightarrow \infty$, and such that for every $i$ there exists a subsequence, possibly depending on $i,\left(\boldsymbol{\nu}_{h}^{i}\right)_{h}$, satisfying

$$
\left(\boldsymbol{\nu}^{j_{h}^{i}}\right)_{t_{i}} \rightarrow \boldsymbol{\nu}_{t_{i}}, \quad 2 \text {-weakly* }
$$

as $h \rightarrow \infty$. Then $(\boldsymbol{\nu}, \boldsymbol{\mu}) \in A Y(A, \boldsymbol{w})$.

Proof. Fix a finite sequence $t_{1}<\ldots<t_{n}$ in $A$. By definition of $A Y\left(\left\{t_{1}, \ldots, t_{n}\right\}, \boldsymbol{w}\right)$, for every $j$ and every $i=1, \ldots, n$, there exist $\left(z_{i}^{j, k}\right)_{k} \in \mathrm{L}^{2}\left(D ; \mathbb{R}^{m}\right)$ and $\left(v_{i}^{j, k}\right)_{k} \in \mathrm{H}_{\Gamma_{0}}^{1}\left(\boldsymbol{w}\left(t_{i}\right)\right)$ satisfying (6.1) for $\boldsymbol{\mu}_{j}$ and such that for every $i$ and $j$ there exists an increasing sequence of integers $\left(k_{l}^{i, j}\right)_{l}$ for which $\left(z_{i}^{j, k_{l}^{i, j}}, v_{i}^{j, k_{l}^{i, j}}\right)_{l}$ satisfies (6.2) for $\boldsymbol{\nu}^{j}$; thanks to Remark 6.5 we can assume, without loss of generality, that

$$
\left\|z_{i}^{j, k}\right\|_{2}^{2} \leq \int_{D \times \mathbb{R}^{m}}|\theta|^{2} \mathrm{~d} \boldsymbol{\mu}_{t_{i}}^{j}(x, \theta)+1, \quad \text { for every } k,
$$

and

$$
\left\|\nabla v_{i}^{j, k_{l}^{i, j}}\right\|_{2}^{2} \leq \int_{D \times \mathbb{R}^{m} \times \mathbb{R}^{N \times d}}|(\theta, F)|^{2} \mathrm{~d} \boldsymbol{\nu}_{t_{i}}^{j}(x, \theta, F)+1, \quad \text { for every } l ;
$$

hence thanks to (6.7) and (6.8) there exists a positive constant $C$ such that

$$
\left\|z_{i}^{j, k}\right\|_{2}^{2} \leq C+1
$$


for every $i, j, k$, and

$$
\sup _{h} \sup _{l}\left\|\nabla v_{i}^{j_{h}^{i}, k_{l}^{i, j} h_{h}^{i}}\right\|_{2}^{2} \leq C+1
$$

for every $i, h, l$. Thanks to Remark 4.1, we can find a metric $d_{1}$ on $Y\left(D ;\left(\mathbb{R}^{m}\right)^{n}\right)$ and a metric $d_{2}$ on $Y\left(D ; \mathbb{R}^{m} \times\right.$ $\mathbb{R}^{N \times d}$ ) which induce the weak* topologies of $Y\left(D ;\left(\mathbb{R}^{m}\right)^{n}\right)$ and $Y\left(D ; \mathbb{R}^{m} \times \mathbb{R}^{N \times d}\right)$, respectively; therefore, for every $j$ we can find an integer $\kappa(j)$ such that, for every $k \geq \kappa(j)$ it holds

$$
d_{1}\left(\delta_{\left(z_{1}^{j, k}, \ldots, z_{n}^{j, k}\right)}, \boldsymbol{\mu}_{t_{1} \ldots t_{n}}^{j}\right)<\frac{1}{j}
$$

analogously, for every $i=1, \ldots, n$, there exists an integer $\kappa_{i}(j)$ such that,

$$
d_{2}\left(\delta_{\left(z_{i}^{j, k} k_{l}^{i, j}, \nabla v_{i}^{j, k_{l}^{i, j}}\right)}, \boldsymbol{\nu}_{t_{i}}^{j}\right)<\frac{1}{j}
$$

whenever $k_{l}^{i, j} \geq \kappa_{i}(j)$.

By taking, if needed, a larger value of $\kappa(j)$, we may assume that (6.11) and (6.12) are satisfied whenever $k \geq \kappa(j)$ and $k_{l}^{i, j} \geq \kappa(j)$, respectively. Another slight modification allows us to assume that, for every $i=1, \ldots, n$ and for every $j$, there exists $k_{l_{i, j}}^{i, j}$ with

$$
\kappa(j)<k_{l_{i, j}}^{i, j} \leq \kappa(j+1)
$$

Let $(\alpha(k))_{k>\kappa(1)}$ be the unique sequence such that $\kappa(\alpha(k))<k \leq \kappa(\alpha(k)+1)$, for every $k>\kappa(1)$. This implies

$$
d_{1}\left(\delta_{\left(z_{1}^{\alpha(k), k}, \ldots, z_{n}^{\alpha(k), k}\right)}, \boldsymbol{\mu}_{t_{1} \ldots t_{n}}^{\alpha(k)}\right) \leq \frac{1}{\alpha(k)}
$$

which, together with (6.7) and (6.9), implies that

$$
\delta_{\left(z_{1}^{\alpha(k), k}, \ldots, z_{n}^{\alpha(k), k}\right)} \rightarrow \boldsymbol{\mu}_{t_{1} \ldots t_{n}}
$$

2 -weakly* as $k \rightarrow \infty$.

Now, for every $i=1, \ldots, n$ we can choose an integer $\beta_{i}(j)$ in such a way that

$$
\beta_{i}(j)=k_{l_{i, j}}^{i, j},
$$

for every $j$, so that we have $\kappa(j)<\beta_{i}(j) \leq \kappa(j+1)$, for every $j$, by (6.13). This implies that $\alpha\left(\beta_{i}(j)\right)=j$ and $\beta_{i}(j)>\kappa(j)$ so that, by $(6.12)$

$$
d_{2}\left(\delta_{\left(z_{i}^{j, \beta_{i}(j)}, \nabla v_{i}^{j, \beta_{i}(j)}\right)}, \boldsymbol{\nu}_{t_{i}}^{j}\right)<\frac{1}{j} .
$$

Therefore, thanks to (6.8) and (6.10) we can conclude that

$$
\delta\left(z_{i}^{\alpha\left(\beta_{i}\left(j_{h}^{i}\right)\right), \beta_{i}\left(j_{h}^{i}\right)}, \nabla v_{i}^{\alpha\left(\beta_{i}\left(j_{h}^{i}\right)\right), \beta_{i}\left(j_{h}^{i}\right)}\right)-\boldsymbol{\nu}_{t_{i}}
$$

as $h \rightarrow \infty$, for every $i=1, \ldots, n$. Since for every $i$ the sequence $\left(\beta_{i}\left(j_{h}^{i}\right)\right)_{h}$ is increasing, (6.15) and (6.17) show that conditions (ap1) and (ap2) in Definition 6.2 are satisfied. 
The following lemma consider the case of varying boundary conditions.

Lemma 6.7. Let $\boldsymbol{w}^{j}$ be a sequence of functions from $A$ into $\mathrm{H}^{1}\left(D, \mathbb{R}^{m}\right)$, such that $\boldsymbol{w}^{j}(t) \rightarrow \boldsymbol{w}(t)$ strongly in $\mathrm{H}^{1}$, for every $t \in A$ and let $(\boldsymbol{\nu}, \boldsymbol{\mu}) \in Y^{2}\left(D ; \mathbb{R}^{m} \times \mathbb{R}^{N \times d}\right)^{A} \times S Y^{2}\left(A, D ; \mathbb{R}^{m}\right)$. Assume that for every finite sequence $t_{1}<\ldots<t_{n}$ in $A$ there exists a sequence $\left(\boldsymbol{\nu}^{j}, \boldsymbol{\mu}^{j}\right) \in A Y\left(\left\{t_{1}, \ldots, t_{n}\right\}, \boldsymbol{w}^{j}\right)$ such that

$$
\boldsymbol{\mu}_{t_{1} \ldots t_{n}}^{j} \rightarrow \boldsymbol{\mu}_{t_{1} \ldots t_{n}} \quad 2-\text { weakly* }
$$

as $j \rightarrow \infty$, and such that for every $i$ there exists a subsequence, possibly depending on $i,\left(\boldsymbol{\nu}_{h}^{i}\right)_{h}$, satisfying

$$
\left(\boldsymbol{\nu}^{j_{h}^{i}}\right)_{t_{i}} \rightarrow \boldsymbol{\nu}_{t_{i}}, \quad 2-w e a k l y^{*}
$$

as $h \rightarrow \infty$. Then $(\boldsymbol{\nu}, \boldsymbol{\mu}) \in A Y(A, \boldsymbol{w})$.

Proof. Fixed $t_{1}<\ldots<t_{n}$ in $[0, T]$, thanks to Lemma 4.5 from (6.19) we can deduce that for every $i=1, \ldots, n$

$$
\tilde{\mathcal{T}}_{\nabla \boldsymbol{w}\left(t_{i}\right)-\nabla \boldsymbol{w}_{h\left(t_{i}\right)}^{2}}\left(\boldsymbol{\nu}^{j_{h}^{i}}\right)_{t_{i}} \rightarrow \boldsymbol{\nu}_{t_{i}} \quad 2 \text {-weakly* }
$$

as $h \rightarrow \infty$, where $\tilde{\mathcal{T}}_{\nabla \boldsymbol{w}\left(t_{i}\right)-\nabla \boldsymbol{w}_{h}^{j_{h}}\left(t_{i}\right)}^{2}$ is the map defined by $\tilde{\mathcal{T}}_{\nabla \boldsymbol{w}\left(t_{i}\right)-\nabla \boldsymbol{w}^{j_{h}^{i}\left(t_{i}\right)}}^{2}(x, \theta, F):=\left(x, \theta, F+\nabla \boldsymbol{w}\left(t_{i}\right)-\right.$ $\left.\nabla \boldsymbol{w}^{j_{h}^{i}}\left(t_{i}\right)\right)$. Thanks to Lemma 4.4 it is easy to see that the hypotheses of Lemma 6.6 are satisfied with $\left(\boldsymbol{\nu}^{j_{h}^{i}}\right)_{t_{i}}$ replaced by $\tilde{\mathcal{T}}_{\nabla \boldsymbol{w}\left(t_{i}\right)-\nabla \boldsymbol{w}_{h}^{j^{i}\left(t_{i}\right)}}^{2}\left(\left(\boldsymbol{\nu}^{j_{h}^{i}}\right)_{t_{i}}\right)$.

Remark 6.8. If $(\boldsymbol{\nu}, \boldsymbol{\mu}) \in A Y(A, \boldsymbol{w})$, for every $t \in A$ there exists a function $\boldsymbol{v}(t) \in \mathrm{H}_{\Gamma_{0}}^{1}(\boldsymbol{w}(t))$ such that $\nabla \boldsymbol{v}(t)=$ $\operatorname{bar}\left(\tilde{\pi}_{\mathbb{R}^{N \times d}}\left(\boldsymbol{\nu}_{t}\right)\right)$. Indeed, by definition of $A Y(A, \boldsymbol{w})$, for every $t \in A$ there exists a sequence $v^{k} \in \mathrm{H}_{\Gamma_{0}}^{1}(\boldsymbol{w}(t))$ such that $\delta_{\nabla v^{k}} \rightarrow \tilde{\pi}_{\mathbb{R}^{N \times d}}\left(\boldsymbol{\nu}_{t}\right), 2$-weakly*; thanks to a variant of Lemma 4.9 with $\mathrm{H}_{0}^{1}$ replaced by $\mathrm{H}_{\Gamma_{0}}^{1}(\boldsymbol{w}(t))$, there exists a function $\boldsymbol{v}(t) \in \mathrm{H}^{1}(D ; \mathbb{R})$, such that $v^{k} \rightarrow \boldsymbol{v}(t)$ weakly in $\mathrm{H}^{1}$ and $\nabla \boldsymbol{v}(t)=\operatorname{bar}\left(\tilde{\pi}_{\mathbb{R}^{N \times d}}\left(\boldsymbol{\nu}_{t}\right)\right)$; since $\mathrm{H}_{\Gamma_{0}}^{1}(\boldsymbol{w}(t))$ is closed with respect to the weak convergence, we can conclude that $\boldsymbol{v}(t) \in \mathrm{H}_{\Gamma_{0}}^{1}(\boldsymbol{w}(t))$.

Translating the previous remark in terms of stochastic processes we obtain the following:

Remark 6.9. If $\left(\boldsymbol{Z}_{t}, \boldsymbol{Y}_{t}\right)_{t \in[0, T]} \in A Y_{s p}([0, T], \boldsymbol{\varphi})$, for every $t \in A$ there exists a function $\boldsymbol{v}(t) \in \mathrm{H}_{\Gamma_{0}}^{1}(\boldsymbol{w}(t))$ such that $\nabla \boldsymbol{v}(t)=\operatorname{bar}\left(\left(\pi_{D}, \boldsymbol{Y}_{t}\right)(P)\right)$.

Remark 6.10. If $(\boldsymbol{\nu}, \boldsymbol{\mu}) \in A Y(A, \boldsymbol{w})$, for every $t \in A$ we define

$$
\boldsymbol{\sigma}(t, x):=\int_{\mathbb{R}^{m} \times \mathbb{R}^{N \times d}} \frac{\partial W}{\partial F}(\theta, F) \mathrm{d} \boldsymbol{\nu}_{t}^{x}(\theta, F),
$$

for a.e. $x \in D$. For every $t \in A$ we have that $\boldsymbol{\sigma}(t) \in \mathrm{L}^{2}\left(D ; \mathbb{R}^{N \times d}\right)$ : this comes immediately from (W.2), (2.2), and from the fact that $\boldsymbol{\nu}_{t} \in Y^{2}\left(D ; \mathbb{R}^{m} \times \mathbb{R}^{N \times d}\right)$. In the language of stochastic processes $\boldsymbol{\sigma}(t)$ can be characterized as the unique element of $\mathrm{L}^{2}\left(D ; \mathbb{R}^{N \times d}\right)$ such that

$$
\int_{D} \boldsymbol{\sigma}(t, x) g(x) \mathrm{d} x=\int_{D \times \Omega} \frac{\partial W}{\partial F}\left(\boldsymbol{Z}_{t}(x, \omega), \boldsymbol{Y}_{t}(x, \omega)\right) g(x) \mathrm{d} P(x, \omega),
$$

for every $g \in \mathrm{L}^{2}\left(D ; \mathbb{R}^{N \times d}\right)$, where $\left(\boldsymbol{Z}_{t}, \boldsymbol{Y}_{t}\right)_{t \in[0, T]}$ is the stochastic process corresponding to $(\boldsymbol{\nu}, \boldsymbol{\mu})$.

Remark 6.11. Since $\varphi \in A C\left([0, T] ; \mathrm{H}^{1}\left(D ; \mathbb{R}^{N}\right)\right)$ and $\boldsymbol{l} \in A C\left([0, T] ; \mathrm{H}^{1}\left(D ; \mathbb{R}^{N}\right)^{*}\right)$, the time derivative $\dot{\varphi}$ and $\boldsymbol{l}$ are well defined for a.e. $t \in[0, T]$ and belong to the space $\mathrm{L}^{1}\left([0, T] ; \mathrm{H}^{1}\left(D ; \mathbb{R}^{N}\right)\right)$ and $\mathrm{L}^{1}\left([0, T] ; \mathrm{H}^{1}\left(D ; \mathbb{R}^{N}\right)^{*}\right)$, respectively. Moreover the fundamental Theorem of Calculus holds (see, e.g., [3], Appendice). 


\subsection{Main result}

We are now in the position to define the notion of globally stable quasistatic evolution of stochastic processes.

Definition 6.12. Given $\varphi \in A C\left([0, T] ; \mathrm{H}^{1}\left(D ; \mathbb{R}^{N}\right)\right), \boldsymbol{l} \in A C\left([0, T] ; \mathrm{H}^{1}\left(D ; \mathbb{R}^{N}\right)^{*}\right), z_{0} \in \mathrm{L}^{2}\left(D ; \mathbb{R}^{m}\right), v_{0} \in \mathcal{A}(0)$, and $T>0$, a globally stable quasistatic evolution of stochastic processes with boundary datum $\varphi$, external load $\boldsymbol{l}$, and initial condition $\left(z_{0}, v_{0}\right)$, in the time interval $[0, T]$, is a stochastic process $\left(\boldsymbol{Z}_{t}, \boldsymbol{Y}_{t}\right)_{t \in[0, T]} \in$ $A Y_{s p}([0, T], \varphi)$, such that for every finite sequence $t_{1}<\ldots<t_{n}$ in $[0, T]$ we have

$$
\left(\pi_{D}, \boldsymbol{Z}_{s_{1}^{j}}, \ldots, \boldsymbol{Z}_{s_{n}^{j}}\right)(P) \rightarrow\left(\pi_{D}, \boldsymbol{Z}_{t_{1}}, \ldots, \boldsymbol{Z}_{t_{n}}\right)(P)
$$

2-weakly*, as $s_{i}^{j} \rightarrow t_{i}$ with $s_{i}^{j} \leq t_{i}$, and satisfying the following conditions:

(ev0) initial condition: $\left(\boldsymbol{Z}_{0}, \boldsymbol{Y}_{0}\right)=\left(z_{0}, \nabla v_{0}\right)$;

(ev1) global stability: for every $t \in[0, T]$, we have

$\int_{D \times \Omega} W\left(\boldsymbol{Z}_{t}(x, \omega), \boldsymbol{Y}_{t}(x, \omega)\right) \mathrm{d} P(x, \omega) \leq$

$$
\int_{D \times \Omega} W\left(\boldsymbol{Z}_{t}(x, \omega)+\tilde{z}(x), \boldsymbol{Y}_{t}(x, \omega)+\nabla \tilde{u}(x)\right) \mathrm{d} P(x, \omega)-\langle\boldsymbol{l}(t), \tilde{u}\rangle+\mathcal{H}(\tilde{z}),
$$

for every $\tilde{z} \in \mathrm{L}^{2}\left(D ; \mathbb{R}^{m}\right)$ and every $\tilde{u} \in \mathrm{H}_{\Gamma_{0}}^{1}(0)$;

(ev2) energy inequality: for every $t \in[0, T]$ we have

$$
\operatorname{Var}_{H}(\boldsymbol{Z}, P ; 0, t):=\sup \sum_{i=1}^{k} \int_{D \times \Omega} H\left(\boldsymbol{Z}_{t_{i}}(x, \omega)-\boldsymbol{Z}_{t_{i-1}}(x, \omega)\right) \mathrm{d} P(x, \omega)<\infty,
$$

where the supremum is taken over all finite partitions $0=t_{0}<\ldots<t_{k}=t$, and the map

$$
t \mapsto[\langle\boldsymbol{\sigma}(t), \nabla \dot{\boldsymbol{\varphi}}(t)\rangle-\langle\dot{\boldsymbol{l}}(t), \boldsymbol{v}(t)\rangle]
$$

is measurable on $[0, T]$, where $\boldsymbol{\sigma}(t)$ is the function defined in (6.21) and $\boldsymbol{v}(t)$ that one defined in Remark 6.9; moreover

$$
\begin{gathered}
\int_{D \times \Omega} W\left(\boldsymbol{Z}_{t}(x, \omega), \boldsymbol{Y}_{t}(x, \omega)\right) \mathrm{d} P(x, \omega)-\langle\boldsymbol{l}(t), \boldsymbol{v}(t)\rangle+\operatorname{Var}_{H}(\boldsymbol{Z}, P ; 0, t) \leq \\
\mathcal{W}\left(z_{0}, v_{0}\right)-\left\langle\boldsymbol{l}(0), v_{0}\right\rangle+\int_{0}^{t}\langle\boldsymbol{\sigma}(s), \nabla \dot{\boldsymbol{\varphi}}(s)\rangle \mathrm{d} s \\
-\int_{0}^{t}[\langle\boldsymbol{l}(s), \dot{\boldsymbol{\varphi}}(s)\rangle+\langle\dot{\boldsymbol{l}}(s), \boldsymbol{v}(s)\rangle] \mathrm{d} s .
\end{gathered}
$$

We now state the main existence theorem in terms of stochastic processes.

Theorem 6.13. Let $\varphi \in A C\left([0, T] ; \mathrm{H}^{1}\left(D ; \mathbb{R}^{N}\right)\right), \boldsymbol{l} \in A C\left([0, T] ; \mathrm{H}^{1}\left(D ; \mathbb{R}^{N}\right)^{*}\right), T>0, z_{0} \in \mathrm{L}^{2}\left(D ; \mathbb{R}^{m}\right)$ and $v_{0} \in \mathcal{A}(0)$ be such that

$$
\mathcal{W}\left(z_{0}, v_{0}\right) \leq \mathcal{W}\left(z_{0}+\tilde{z}, v_{0}+\tilde{u}\right)-\langle\boldsymbol{l}(0), \tilde{u}\rangle+\mathcal{H}(\tilde{z})
$$

for every $\tilde{z} \in \mathrm{L}^{2}\left(D ; \mathbb{R}^{m}\right)$ and every $\tilde{u} \in \mathrm{H}_{\Gamma_{0}}^{1}(0)$. Then there exists a globally stable quasistatic evolution for stochastic processes with boundary datum $\boldsymbol{\varphi}$, external load $\boldsymbol{l}$, and initial condition $\left(z_{0}, v_{0}\right)$, in the time interval $[0, T]$. 
Thanks to Remark 6.4, we can translate the definition of globally stable quasistatic evolution of stochastic processes in terms of globally stable quasistatic evolution of Young measures.

Definition 6.14. Given $\varphi \in A C\left([0, T] ; \mathrm{H}^{1}\left(D ; \mathbb{R}^{N}\right)\right), \boldsymbol{l} \in A C\left([0, T] ; \mathrm{H}^{1}\left(D ; \mathbb{R}^{N}\right)^{*}\right), z_{0} \in \mathrm{L}^{2}\left(D ; \mathbb{R}^{m}\right), v_{0} \in$ $\mathcal{A}(0)$, and $T>0$, a globally stable quasistatic evolution of Young measures with boundary datum $\varphi$, external load $\boldsymbol{l}$ and initial condition $\left(z_{0}, v_{0}\right)$, in the time interval $[0, T]$, is a pair $(\boldsymbol{\nu}, \boldsymbol{\mu}) \in A Y([0, T], \boldsymbol{\varphi})$, with $\boldsymbol{\mu} \in$ $S Y_{-}^{2}\left([0, T], D ; \mathbb{R}^{m}\right)$, satisfying the following conditions:

(ev0) initial condition: $\boldsymbol{\nu}_{0}=\delta_{\left(z_{0}, \nabla v_{0}\right)}$;

(ev1) global stability: for every $t \in[0, T]$, we have

$\int_{D \times \mathbb{R}^{m} \times \mathbb{R}^{N \times d}} W(\theta, F) \mathrm{d} \boldsymbol{\nu}_{t}(x, \theta, F) \leq$

$$
\int_{D \times \mathbb{R}^{m} \times \mathbb{R}^{N \times d}} W(\theta+\tilde{z}(x), F+\nabla \tilde{u}(x)) \mathrm{d} \boldsymbol{\nu}_{t}(x, \theta, F)-\langle\boldsymbol{l}(t), \tilde{u}\rangle+\mathcal{H}(\tilde{z}),
$$

for every $\tilde{z} \in \mathrm{L}^{2}\left(D ; \mathbb{R}^{m}\right)$ and every $\tilde{u} \in \mathrm{H}_{\Gamma_{0}}^{1}(0)$;

(ev2) energy inequality: for every $t \in[0, T]$ we have that $\operatorname{Var}_{H}(\boldsymbol{\mu} ; 0, t)<\infty$, (see (4.9)), and the map

$$
t \mapsto[\langle\boldsymbol{\sigma}(t), \nabla \dot{\boldsymbol{\varphi}}(t)\rangle-\langle\dot{\boldsymbol{l}}(t), \boldsymbol{v}(t)\rangle]
$$

is measurable on $[0, T]$, where $\boldsymbol{\sigma}(t)$ is the function defined in (6.20) and $\boldsymbol{v}(t)$ that one defined in Remark 6.8; moreover

$$
\begin{aligned}
\int_{D \times \mathbb{R}^{m} \times \mathbb{R}^{N \times d}} W(\theta, F) \mathrm{d} \boldsymbol{\nu}_{t}(x, \theta, F)-\langle\boldsymbol{l}(t), \boldsymbol{v}(t)\rangle+\operatorname{Var}_{H}(\boldsymbol{\mu} ; 0, t) \leq \\
\mathcal{W}\left(z_{0}, v_{0}\right)-\left\langle\boldsymbol{l}(0), v_{0}\right\rangle+\int_{0}^{t}\langle\boldsymbol{\sigma}(s), \nabla \dot{\boldsymbol{\varphi}}(s)\rangle \mathrm{d} s-\int_{0}^{t}[\langle\boldsymbol{l}(s), \dot{\boldsymbol{\varphi}}(s)\rangle+\langle\dot{\boldsymbol{l}}(s), \boldsymbol{v}(s)\rangle] \mathrm{d} s .
\end{aligned}
$$

Thanks to Theorem 3.5, to obtain the main theorem it is enough to prove the following version for Young measures.

Theorem 6.15. Let $\varphi \in A C\left([0, T] ; \mathrm{H}^{1}\left(D ; \mathbb{R}^{N}\right)\right), \boldsymbol{l} \in A C\left([0, T] ; \mathrm{H}^{1}\left(D ; \mathbb{R}^{N}\right)^{*}\right), T>0, z_{0} \in \mathrm{L}^{2}\left(D ; \mathbb{R}^{m}\right)$, and $v_{0} \in \mathcal{A}(0)$ be such that

$$
\mathcal{W}\left(z_{0}, v_{0}\right) \leq \mathcal{W}\left(z_{0}+\tilde{z}, v_{0}+\tilde{u}\right)-\langle\boldsymbol{l}(0), \tilde{u}\rangle+\mathcal{H}(\tilde{z}),
$$

for every $\tilde{z} \in \mathrm{L}^{2}\left(D ; \mathbb{R}^{m}\right)$ and every $\tilde{u} \in \mathrm{H}_{\Gamma_{0}}^{1}(0)$. Then there exists a globally stable quasistatic evolution for Young measures with boundary datum $\boldsymbol{\varphi}$, external load $\boldsymbol{l}$, and initial condition $\left(z_{0}, v_{0}\right)$, in the time interval $[0, T]$.

Remark 6.16. In the proof of Theorem 6.15 we will obtain, in particular, a globally stable quasistatic evolution $(\boldsymbol{\nu}, \boldsymbol{\mu})$ and a subset $\Theta$ of $[0, T]$ which satisfy the following property:

(appr) for every $t \in[0, T]$ there exists a sequence $s^{j}$ in $\Theta$ with $s^{j} \leq t$ and $s^{j} \rightarrow t$, such that

$$
\nu_{s^{j}} \rightarrow \nu_{t} \quad \text { 2-weakly*. }
$$

\section{Proof OF THE MAIN THEOREM}

The proof is obtained via time discretization, resolution of incremental minimum problems, and passing to the limit as the discretization step tends to 0 . 


\subsection{The incremental minimum problem}

Let us fix a sequence of subdivisions of $[0, T], 0=t_{n}^{0}<t_{n}^{1}<\ldots<t_{n}^{k(n)}=T$, such that $\sup _{i=1, \ldots, k(n)} \tau_{n}^{i} \rightarrow 0$, as $n \rightarrow \infty$, where $\tau_{n}^{i}:=t_{n}^{i}-t_{n}^{i-1}$, for every $i=1, \ldots, k(n)$.

For every $i=0,1, \ldots, k(n)$ we set $\boldsymbol{l}_{n}^{i}:=\boldsymbol{l}\left(t_{n}^{i}\right)$ and $\boldsymbol{\varphi}_{n}^{i}:=\boldsymbol{\varphi}\left(t_{n}^{i}\right)$.

We will define $\left(\boldsymbol{\nu}_{n}^{i}, \boldsymbol{\mu}_{n}^{i}\right) \in A Y\left(\left\{t_{n}^{0}, \ldots, t_{n}^{i}\right\}, \boldsymbol{\varphi}\right)$ by induction on $i$ : set $\left(\boldsymbol{\nu}_{n}^{0}, \boldsymbol{\mu}_{n}^{0}\right):=\delta_{\left(z_{0}, \nabla v_{0}\right)}$, and for $i>0$ we define $\left(\boldsymbol{\nu}_{n}^{i}, \boldsymbol{\mu}_{n}^{i}\right)$ as a minimizer (see Lem. 7.2 below) of the functional

$$
\int_{D \times \mathbb{R}^{m} \times \mathbb{R}^{N \times d}} W(\theta, F) \mathrm{d} \boldsymbol{\nu}_{t_{n}^{i}}(x, \theta, F)-\left\langle\boldsymbol{l}_{n}^{i}, \boldsymbol{v}\left(t_{n}^{i}\right)\right\rangle+\int_{D \times\left(\mathbb{R}^{m}\right)^{2}} H\left(\theta_{i}-\theta_{i-1}\right) \mathrm{d} \boldsymbol{\mu}_{t_{n}^{i-1}} t_{n}^{i}\left(x, \theta_{i-1}, \theta_{i}\right),
$$

in the set $A_{n}^{i}$ of all $(\boldsymbol{\nu}, \boldsymbol{\mu}) \in A Y\left(\left\{t_{n}^{0}, \ldots, t_{n}^{i}\right\}, \boldsymbol{\varphi}\right)$, satisfying

$$
\begin{gathered}
\boldsymbol{\mu}_{t_{n}^{0} \ldots t_{n}^{i-1}}=\left(\boldsymbol{\mu}_{n}^{i-1}\right)_{t_{n}^{0} \ldots t_{n}^{i-1}} \\
\boldsymbol{\nu}_{t_{n}^{j}}=\left(\boldsymbol{\nu}_{n}^{i-1}\right)_{t_{n}^{j}}, \quad \text { for every } j<i,
\end{gathered}
$$

where the function $\boldsymbol{v}\left(t_{n}^{i}\right)$ appearing in (7.1) is that one defined in Remark 6.8.

Lemma 7.1. The set $A_{n}^{i}$ is nonempty, for every $i>1$.

Proof. Fixed $\left(\boldsymbol{\nu}_{n}^{i-1}, \boldsymbol{\mu}_{n}^{i-1}\right)$, we consider the map $\tilde{\mathcal{T}}_{\nabla \boldsymbol{\varphi}_{n}^{i}-\nabla \boldsymbol{\varphi}_{n}^{i-1}}^{2}:(x, \theta, F) \mapsto\left(x, \theta, F+\nabla \boldsymbol{\varphi}_{n}^{i}(x)-\nabla \boldsymbol{\varphi}_{n}^{i-1}(x)\right)$, and the map $\pi^{(i)}: D \times\left(\mathbb{R}^{m}\right)^{i} \rightarrow D \times\left(\mathbb{R}^{m}\right)^{i+1}$ defined by $\pi^{(i)}\left(x, \theta_{1}, \ldots, \theta_{i-1}\right):=\left(x, \theta_{1}, \ldots, \theta_{i-1}, \theta_{i-1}\right)$; let $\boldsymbol{\nu} \in Y^{2}\left(D ; \mathbb{R}^{m}\right)^{\left\{t_{n}^{0}, \ldots, t_{n}^{i}\right\}}$ be defined by $\boldsymbol{\nu}_{t_{n}^{j}}:=\left(\boldsymbol{\nu}_{n}^{i-1}\right)_{t_{n}^{j}}$, for $j<i$ and $\boldsymbol{\nu}_{t_{n}^{i}}:=\tilde{\mathcal{T}}_{\nabla \boldsymbol{\varphi}_{n}^{i}-\nabla \boldsymbol{\varphi}_{n}^{i-1}}\left(\left(\boldsymbol{\nu}_{n}^{i-1}\right)_{t_{n}^{i-1}}\right)$ and $\boldsymbol{\mu}$

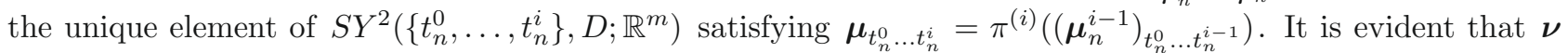
and $\boldsymbol{\mu}$ so defined satisfy the projection properties (7.2) and (7.3). Moreover it is easy to prove that $(\boldsymbol{\nu}, \boldsymbol{\mu}) \in$ $A Y\left(\left\{t_{n}^{0}, \ldots, t_{n}^{i}\right\}, \boldsymbol{\varphi}\right)$ : since $\pi^{(i)}\left(\delta_{\left(z^{0}, \ldots, z^{i-1}\right)}\right)=\delta_{\left(z^{0}, \ldots, z^{i-1}, z^{i-1}\right)}$ and $\tilde{\mathcal{T}}_{\nabla \boldsymbol{\varphi}_{n}^{i}-\nabla \boldsymbol{\varphi}_{n}^{i-1}}\left(\delta_{(z, \nabla v)}\right)=\delta_{\left(z, \nabla v+\nabla \boldsymbol{\varphi}_{n}^{i}-\nabla \boldsymbol{\varphi}_{n}^{i-1}\right)}$, with $v+\varphi_{n}^{i}-\varphi_{n}^{i-1} \in \mathcal{A}\left(t_{n}^{i}\right)$ whenever $v \in \mathcal{A}\left(t_{n}^{i-1}\right)$, applying Lemma 4.4 we can obtain the approximation properties (6.1) and (6.2).

Lemma 7.2. For every $i$ the functional (7.1) has a minimizer over $A_{n}^{i}$.

Proof. Let $\left(\boldsymbol{\nu}^{h}, \boldsymbol{\mu}^{h}\right)_{h} \subset A_{n}^{i}$ be a minimizing sequence. By the bounds on $W$ and the assumption on $\boldsymbol{l}$, using Poincaré inequality we have

$$
\begin{aligned}
& c \int_{D \times \times \mathbb{R}^{N \times \mathrm{d}}}\left[|\theta|^{2}+|F|^{2}\right] \mathrm{d} \boldsymbol{\nu}_{t_{n}^{i}}^{h}(x, \theta, F)-C^{\prime}\left(1+\left\|\nabla \boldsymbol{v}^{h}\left(t_{n}^{i}\right)\right\|_{2}\right) \leq \\
& \qquad \int_{D \times \mathbb{R}^{m} \times \mathbb{R}^{N \times d}} W(\theta, F) \mathrm{d} \boldsymbol{\nu}_{t_{n}^{i}}^{h}(x, \theta, F)-\left\langle\boldsymbol{l}_{n}^{i}, \boldsymbol{v}^{h}\left(t_{n}^{i}\right)\right\rangle \leq C^{\prime},
\end{aligned}
$$

for every $h$, for positive constants $c, C^{\prime}$. Since by Remark 6.8

$$
\left\|\nabla \boldsymbol{v}^{h}\left(t_{n}^{i}\right)\right\|_{2} \leq\left(\int_{D \times \mathbb{R}^{m} \times \mathbb{R}^{N \times d}}|F|^{2} \mathrm{~d} \boldsymbol{\nu}_{t_{n}^{i}}^{h}(x, \theta, F)\right)^{1 / 2},
$$

we can deduce that

$$
\sup _{h} \int_{D \times \mathbb{R}^{m} \times \mathbb{R}^{N \times d}}|(\theta, F)|^{2} \mathrm{~d} \boldsymbol{\nu}_{t_{n}^{i}}^{h}(x, \theta, F) \leq C^{\prime} .
$$


Since, thanks to Remark 6.3

$$
\begin{aligned}
\int_{D \times\left(\mathbb{R}^{m}\right)^{i+1}}\left|\left(\theta_{0}, \ldots, \theta_{i}\right)\right|^{2} \mathrm{~d} \boldsymbol{\mu}_{t_{n}^{0} \ldots t_{n}^{i}}^{h}\left(x, \theta_{0}, \ldots, \theta_{i}\right) & =\sum_{j=0}^{i+1} \int_{D \times \mathbb{R}^{m}}\left|\theta_{j}\right|^{2} \mathrm{~d} \boldsymbol{\mu}_{t_{n}^{j}}^{h}\left(x, \theta_{j}\right) \\
& =\sum_{j=0}^{i+1} \int_{D \times \mathbb{R}^{m} \times \mathbb{R}^{N \times d}}\left|\theta_{j}\right|^{2} \mathrm{~d} \boldsymbol{\nu}_{t_{n}^{j}}^{h}\left(x, F, \theta_{j}\right),
\end{aligned}
$$

the projection property (7.3) and (7.5) imply that the second moments of $\boldsymbol{\mu}_{t_{n}^{0} \ldots t_{n}^{i}}^{h}$ are bounded uniformly with respect to $h$. From this and from $(7.5)$ we can deduce that, up to a subsequence, there exist $\bar{\mu} \in Y^{2}\left(D ;\left(\mathbb{R}^{m}\right)^{i+1}\right)$ and $\bar{\nu} \in Y^{2}\left(D ; \mathbb{R}^{m} \times \mathbb{R}^{N \times d}\right)$ such that

$$
\begin{gathered}
\boldsymbol{\mu}_{t_{n}^{0} \ldots t_{n}^{i}}^{h} \rightarrow \bar{\mu} \quad 2 \text {-weakly* } \\
\boldsymbol{\nu}_{t_{n}^{i}}^{h} \rightarrow \bar{\nu} \quad \text { 2-weakly* }
\end{gathered}
$$

Hence defining

$$
\begin{gathered}
\overline{\boldsymbol{\nu}}_{t_{n}^{j}}:=\left(\boldsymbol{\nu}_{n}^{i-1}\right)_{t_{n}^{j}}, \quad \text { for every } j<i \\
\overline{\boldsymbol{\nu}}_{t_{n}^{i}}:=\bar{\nu}
\end{gathered}
$$

and $\overline{\boldsymbol{\mu}}$ as the unique element of $S Y^{2}\left(\left\{t_{n}^{0}, \ldots, t_{n}^{i}\right\}, D ; \mathbb{R}^{m}\right)$ such that $\overline{\boldsymbol{\mu}}_{t_{n}^{0} \ldots t_{n}^{i}}=\bar{\mu}$, we obtain

$$
(\overline{\boldsymbol{\nu}}, \overline{\boldsymbol{\mu}}) \in Y^{2}\left(D ; \mathbb{R}^{m} \times \mathbb{R}^{N \times d}\right)^{\left\{t_{n}^{0}, \ldots, t_{n}^{i}\right\}} \times S Y^{2}\left(\left\{t_{n}^{0}, \ldots, t_{n}^{i}\right\}, D ; \mathbb{R}^{m}\right)
$$

Since the hypotheses of Lemma 6.6 are satisfied, we deduce that $(\overline{\boldsymbol{\nu}}, \overline{\boldsymbol{\mu}}) \in A Y\left(\left\{t_{n}^{0}, \ldots, t_{n}^{i}\right\}, \boldsymbol{\varphi}\right)$. Moreover, by construction, $(\overline{\boldsymbol{\nu}}, \overline{\boldsymbol{\mu}})$ satisfies also the projection properties $(7.2)$ and $(7.3)$, so we can conclude that $(\overline{\boldsymbol{\nu}}, \overline{\boldsymbol{\mu}}) \in A_{n}^{i}$.

By (4.1) the terms of (7.1) containing $W$ and $H$ are lower semicontinuous with respect to the 2 -weak* convergence; on the other hand a variant of Lemma 4.9, with $\mathrm{H}_{0}^{1}$ replaced by $\mathcal{A}\left(t_{n}^{i}\right)$, shows that the term of (7.1) containing $\boldsymbol{l}_{n}^{i}$ is continuous with respect to the 2 -weak* convergence, therefore the functional (7.1) is 2 -weakly* lower semicontinuous and this implies that $(\overline{\boldsymbol{\nu}}, \overline{\boldsymbol{\mu}})$ is a minimizer for it in $A_{n}^{i}$.

Remark 7.3. Even if $W(\theta, \cdot): \mathbb{R}^{N \times d} \rightarrow[0,+\infty)$ is convex for every $\theta \in \mathbb{R}^{m}$, it may happen that the incremental minimum problems have no solutions representable by functions.

We give an example in which this happens even for the first time step. By definition of $A_{n}^{1}$, there exists a solution of the first incremental minimum problem representable by function if and only if there exist $z_{1} \in$ $\mathrm{L}^{2}\left(D ; \mathbb{R}^{m}\right)$ and $v_{1} \in \mathcal{A}\left(t_{n}^{1}\right)$ such that $\left(\left(\delta_{\left(z_{0}, \nabla v_{0}\right)}, \delta_{\left(z_{1}, \nabla v_{1}\right)}\right), \delta_{\left(z_{0}, z_{1}\right)}\right)$ realizes the minimum of the functional (7.1) on $A_{n}^{1}$. Consider the following case: $D=(0,1)^{2}$ and $N=m=1, T=1, \boldsymbol{l} \equiv 0$ and $\varphi\left(t,\left(x_{1}, x_{2}\right)\right):=(1-t) x_{1}$, for every $t \in[0,1]$ and every $\left(x_{1}, x_{2}\right) \in(0,1)^{2}$. We consider

$$
W\left(\theta,\left(F_{1}, F_{2}\right)\right):=\left|F_{1}-a(\theta)\right|^{2}+\left|F_{2}\right|^{2}+b(\theta)+c
$$

where $a$ is a $\mathrm{C}^{1}$ function satisfying $a(0)=a(1)=1$ and $a(-1)=-1$, while $b$ a $\mathrm{C}^{1}$ function such that $\tilde{b}(\theta):=b(\theta)+|\theta|$ is positive and vanishes only at 0,1 , and -1 , and $c:=-\inf b$. It can be easily verified that (W.1) and (W.2) are satisfied by suitable choices of $a$ and $b$ compatible with the requirements above. Now choose $H(\theta):=|\theta|, z_{0} \equiv 0$ and $v_{0}\left(x_{1}, x_{2}\right):=x_{1}$. It is immediate to check that $\left(z_{0}, v_{0}\right)$ satisfy the boundary conditions and (6.24). Moreover, by standard arguments, it can be easily shown that the infimum of functional in (7.1), for $i=1$, is $c$ and cannot be attained by functions which satisfy the boundary conditions. A minimizer 
of (7.1) on $A_{n}^{1}$ is defined by

$$
\begin{aligned}
\left(\boldsymbol{\nu}_{n}^{1}\right)_{t_{n}^{1}} & :=\frac{t_{n}^{1}}{2} \delta_{(-1,(-1,0))}+\left(1-\frac{t_{n}^{1}}{2}\right) \delta_{(1,(1,0))} \\
\left(\boldsymbol{\mu}_{n}^{1}\right)_{t_{n}^{0}, t_{n}^{1}} & :=\frac{t_{n}^{1}}{2} \delta_{(0,-1)}+\left(1-\frac{t_{n}^{1}}{2}\right) \delta_{(0,1)}
\end{aligned}
$$

Set $\tau^{n}(s):=t_{n}^{i}$, whenever $t_{n}^{i} \leq s<t_{n}^{i+1}$, where we set $t_{n}^{k(n)+1}:=T+\frac{1}{n}$.

For every $i$ and $n$ we set

$$
\sigma_{n}^{i}(x):=\int_{D \times \mathbb{R}^{m} \times \mathbb{R}^{N \times d}} \frac{\partial W}{\partial F}(\theta, F) \mathrm{d}\left(\boldsymbol{\nu}_{n}^{i}\right)_{t_{n}^{i}}^{x},
$$

and define

$$
\boldsymbol{\sigma}_{n}(t, x):=\sigma_{n}^{i}(x)
$$

for a.e. $x \in D$, whenever $t_{n}^{i} \leq t<t_{n}^{i+1}$.

We define $\boldsymbol{\nu}_{n} \in Y^{2}\left(D ; \mathbb{R}^{m} \times \mathbb{R}^{N \times d}\right)^{[0, T]}$ by

$$
\left(\boldsymbol{\nu}_{n}\right)_{s}:=\left(\boldsymbol{\nu}_{n}^{i}\right)_{t_{n}^{i}}
$$

whenever $t_{n}^{i}=\tau^{n}(s)$, for every $s \in[0, T]$; we define also $\boldsymbol{\mu}_{n} \in S Y^{2}\left([0, T], D ; \mathbb{R}^{m}\right)$ as the piecewise constant interpolation of $\boldsymbol{\mu}_{n}^{k(n)}$, as in Definition 4.12 .

Note that $\left(\boldsymbol{\nu}_{n}, \boldsymbol{\mu}_{n}\right) \in A Y\left([0, T], \boldsymbol{\varphi}\left(\tau^{n}(\cdot)\right)\right)$ by construction.

\subsection{A priori estimates}

First of all we want to deduce a discrete version of the energy inequality for $\left(\boldsymbol{\nu}_{n}, \boldsymbol{\mu}_{n}\right)$.

Using the competitor defined in the proof of Lemma 7.1 and the fact that

$$
\operatorname{bar}\left(\tilde{\pi}_{\mathbb{R}^{N \times d}}\left(\tilde{\mathcal{T}}_{\nabla \boldsymbol{\varphi}_{n}^{i}-\nabla \boldsymbol{\varphi}_{n}^{i-1}}^{2}\left(\left(\boldsymbol{\nu}_{n}^{i}\right)_{t_{n}^{i}}\right)\right)\right)=\operatorname{bar}\left(\tilde{\pi}_{\mathbb{R}^{N \times d}}\left(\left(\boldsymbol{\nu}_{n}^{i}\right)_{t_{n}^{i}}\right)\right)+\nabla \boldsymbol{\varphi}_{n}^{i}-\nabla \boldsymbol{\varphi}_{n}^{i-1}
$$

for every $i$ and $n$, we have

$$
\begin{array}{r}
\int_{D \times \mathbb{R}^{m} \times \mathbb{R}^{N \times d}} W(\theta, F) \mathrm{d}\left(\boldsymbol{\nu}_{n}^{i}\right)_{t_{n}^{i}}(x, \theta, F)-\left\langle\boldsymbol{l}_{n}^{i}, \boldsymbol{v}_{n}^{i}\left(t_{n}^{i}\right)\right\rangle+\int_{D \times\left(\mathbb{R}^{m}\right)^{2}} H\left(\theta_{i}-\theta_{i-1}\right) \mathrm{d}\left(\boldsymbol{\mu}_{n}^{i}\right)_{t_{n}^{i-1} t_{n}^{i}}\left(x, \theta_{i-1}, \theta_{i}\right) \leq \\
\int_{D \times \mathbb{R}^{m} \times \mathbb{R}^{N \times d}} W\left(\theta, F+\nabla \boldsymbol{\varphi}_{n}^{i}(x)-\nabla \boldsymbol{\varphi}_{n}^{i-1}(x)\right) \mathrm{d}\left(\boldsymbol{\nu}_{n}^{i-1}\right)_{t_{n}^{i-1}}(x, \theta, F)-\left\langle\boldsymbol{l}_{n}^{i}, \boldsymbol{v}_{n}^{i}\left(t_{n}^{i-1}\right)+\boldsymbol{\varphi}_{n}^{i}-\boldsymbol{\varphi}_{n}^{i-1}\right\rangle \\
+\int_{D \times\left(\mathbb{R}^{m}\right)^{i+1}} H\left(\theta_{i}-\theta_{i-1}\right) d\left(\pi ^ { ( i ) } \left(\left(\boldsymbol{\mu}_{n}^{i-1}\right)_{\left.\left.t_{n}^{0} \ldots t_{n}^{i-1}\right)\right)\left(x, \theta_{0}, \ldots, \theta_{i-1}, \theta_{i}\right) .}\right.\right.
\end{array}
$$

We deduce that

$$
\begin{gathered}
\int_{D \times \mathbb{R}^{m} \times \mathbb{R}^{N \times d}} W(\theta, F) \mathrm{d}\left(\boldsymbol{\nu}_{n}^{i}\right)_{t_{n}^{i}}(x, \theta, F)-\left\langle\boldsymbol{l}_{n}^{i}, \boldsymbol{v}_{n}^{i}\left(t_{n}^{i}\right)\right\rangle+\int_{D \times\left(\mathbb{R}^{m}\right)^{2}} H\left(\theta_{i}-\theta_{i-1}\right) \mathrm{d}\left(\boldsymbol{\mu}_{n}^{i}\right)_{t_{n}^{i-1} t_{n}^{i}}\left(x, \theta_{i-1}, \theta_{i}\right) \leq \\
\int_{D \times \mathbb{R}^{m} \times \mathbb{R}^{N \times d}} W(\theta, F) \mathrm{d}\left(\boldsymbol{\nu}_{n}^{i-1}\right)_{t_{n}^{i-1}}(x, \theta, F)-\left\langle\boldsymbol{l}_{n}^{i-1}, \boldsymbol{v}_{n}^{i-1}\left(t_{n}^{i-1}\right)\right\rangle \\
+\int_{D \times \mathbb{R}^{m} \times \mathbb{R}^{N \times d}}\left[W\left(\theta, F+\nabla \boldsymbol{\varphi}_{n}^{i}(x)-\nabla \boldsymbol{\varphi}_{n}^{i-1}(x)\right)-W(\theta, F)\right] \mathrm{d}\left(\boldsymbol{\nu}_{n}^{i-1}\right)_{t_{n}^{i-1}}(x, \theta, F) \\
-\left\langle\boldsymbol{l}_{n}^{i}, \boldsymbol{v}_{n}^{i-1}\left(t_{n}^{i-1}\right)+\boldsymbol{\varphi}_{n}^{i}-\boldsymbol{\varphi}_{n}^{i-1}\right\rangle+\left\langle\boldsymbol{l}_{n}^{i-1}, \boldsymbol{v}_{n}^{i-1}\left(t_{n}^{i-1}\right)\right\rangle .
\end{gathered}
$$


Let us fix $t \in[0, T]$ such that $t_{n}^{j} \leq t<t_{n}^{j+1}$; using

$$
\begin{aligned}
& \int_{D \times \mathbb{R}^{m} \times \mathbb{R}^{N \times d}}\left[W\left(\theta, F+\nabla \boldsymbol{\varphi}_{n}^{i}(x)-\nabla \boldsymbol{\varphi}_{n}^{i-1}(x)\right)-W(\theta, F)\right] \mathrm{d}\left(\boldsymbol{\nu}_{n}^{i-1}\right)_{t_{n}^{i-1}}(x, \theta, F)= \\
& \int_{t_{n}^{i-1}}^{t_{n}^{i}}\left(\int_{D \times \mathbb{R}^{m} \times \mathbb{R}^{N \times d}} \frac{\partial W}{\partial F}\left(\theta, F+\varepsilon^{n}(s, x)\right) \nabla \dot{\boldsymbol{\varphi}}(s, x) \mathrm{d}\left(\boldsymbol{\nu}_{n}\right)_{s}(x, \theta, F)\right) \mathrm{d} s,
\end{aligned}
$$

where $\varepsilon^{n}(s, x):=\nabla \varphi(s, x)-\nabla \varphi\left(\tau^{n}(s), x\right)$, for every $s \in[0, T]$ and every $x \in D$, and

$$
\left\langle\boldsymbol{l}_{n}^{i}, \boldsymbol{v}_{n}^{i-1}\left(t_{n}^{i-1}\right)+\varphi_{n}^{i}-\boldsymbol{\varphi}_{n}^{i-1}\right\rangle-\left\langle\boldsymbol{l}_{n}^{i-1}, \boldsymbol{v}_{n}^{i-1}\left(t_{n}^{i-1}\right)\right\rangle=\int_{t_{n}^{i-1}}^{t_{n}^{i}}\left[\langle\boldsymbol{l}(s), \dot{\varphi}(s)\rangle+\left\langle\boldsymbol{l}(s), \boldsymbol{v}_{n}^{i-1}\left(t_{n}^{i-1}\right)-\boldsymbol{\varphi}\left(\tau^{n}(s)\right)+\boldsymbol{\varphi}(s)\right\rangle\right] \mathrm{d} s,
$$

and iterating from 0 to $j$, we obtain

$$
\begin{aligned}
\int_{D \times \mathbb{R}^{m} \times \mathbb{R}^{N \times d}} W(\theta, F) \mathrm{d}\left(\boldsymbol{\nu}_{n}\right)_{t}(x, \theta, F)-\left\langle\boldsymbol{l}\left(\tau^{n}(t)\right), \boldsymbol{v}_{n}(t)\right\rangle+\operatorname{Var}_{H}\left(\boldsymbol{\mu}_{n} ; 0, t\right) \leq \\
\mathcal{W}\left(z_{0}, v_{0}\right)-\left\langle\boldsymbol{l}(0), v_{0}\right\rangle+\int_{0}^{\tau^{n}(t)}\left\langle\boldsymbol{\sigma}_{n}(s), \nabla \dot{\boldsymbol{\varphi}}(s)\right\rangle \mathrm{d} s-\int_{0}^{\tau^{n}(t)}\left[\left\langle\dot{\boldsymbol{l}}(s), \boldsymbol{v}_{n}(s)\right\rangle+\langle\boldsymbol{l}(s), \dot{\boldsymbol{\varphi}}(s)\rangle\right] \mathrm{d} s \\
+\int_{0}^{\tau^{n}(t)}\left(\int_{D \times \mathbb{R}^{m} \times \mathbb{R}^{N \times d}}\left[\frac{\partial W}{\partial F}\left(\theta, F+\varepsilon^{n}(s, x)\right)-\frac{\partial W}{\partial F}(\theta, F)\right] \nabla \dot{\boldsymbol{\varphi}}(s) \mathrm{d}\left(\boldsymbol{\nu}_{n}\right)_{s}(x, \theta, F)\right) \mathrm{d} s \\
\quad+\int_{0}^{\tau^{n}(t)}\left\langle\dot{\boldsymbol{l}}(s), \boldsymbol{\varphi}\left(\tau^{n}(s)\right)-\boldsymbol{\varphi}(s)\right\rangle \mathrm{d} s
\end{aligned}
$$

From (7.11), we can deduce the following a priori estimates on $\left(\boldsymbol{\nu}_{n}, \boldsymbol{\mu}_{n}\right)$.

Lemma 7.4. There exists a positive constant $C$, such that

$$
\begin{gathered}
\sup _{n} \sup _{t \in[0, T]} \int_{D \times \mathbb{R}^{m} \times \mathbb{R}^{N \times d}}|(\theta, F)|^{2} \mathrm{~d}\left(\boldsymbol{\nu}_{n}\right)_{t}(x, \theta, F) \leq C, \\
\sup _{n} \operatorname{Var}_{H}\left(\boldsymbol{\mu}_{n} ; 0, T\right) \leq C .
\end{gathered}
$$

Proof. Using the fact that $\sup _{t \in[0, T]}\|\boldsymbol{l}(t)\|_{\left(\mathrm{H}^{1}\right)^{*}}, \int_{0}^{T}\|\dot{\boldsymbol{l}}(t)\|_{\left(\mathrm{H}^{1}\right)^{*}} \mathrm{~d} t$, and $\int_{0}^{T}\|\dot{\boldsymbol{\varphi}}(t)\|_{\mathrm{H}^{1}} \mathrm{~d} t$ are finite, the hypotheses on $W$ and the inequality

$$
\sup _{s \in[0, T]} \int_{D \times \mathbb{R}^{m} \times \mathbb{R}^{N \times d}}|(\theta, F)|^{2} \mathrm{~d}\left(\boldsymbol{\nu}_{n}\right)_{s}(x, \theta, F)<\infty
$$

(since $\boldsymbol{\nu}_{n}$ are piecewise constant interpolations of Young measures with finite second moments) we can deduce from (7.11) that, for $n$ sufficiently large,

$$
\int_{D \times \mathbb{R}^{m} \times \mathbb{R}^{N \times d}}|(\theta, F)|^{2} \mathrm{~d}\left(\boldsymbol{\nu}_{n}\right)_{t}(x, \theta, F) \leq \tilde{C}+\tilde{C} \sup _{s \in[0, T]}\left(1+\tilde{c} \int_{D \times \mathbb{R}^{m} \times \mathbb{R}^{N \times d}}|(\theta, F)|^{2} \mathrm{~d}\left(\boldsymbol{\nu}_{n}\right)_{s}(x, \theta, F)\right)^{1 / 2}
$$

for suitable positive constants $\tilde{C}$ and $\tilde{c}$ independent of $t$ and $n$ (to estimate the terms in the third line in (7.11) we use (7.4), while the term in the fourth line of (7.11) can be treated using $\pi_{D}\left(\left(\boldsymbol{\nu}_{n}\right)_{s}\right)=\mathcal{L}^{d}$ and Hölder inequality).

Since this can be repeated for every $t \in[0, T]$, we deduce (7.12). Inequality (7.13) comes now from (7.12) and (7.11). 
We can also deduce the following energy inequality for $\left(\boldsymbol{\nu}_{n}, \boldsymbol{\mu}_{n}\right)$.

Lemma 7.5. For every $t \in[0, T]$ we have

$$
\begin{aligned}
& \int_{D \times \mathbb{R}^{m} \times \mathbb{R}^{N \times d}} W(\theta, F) \mathrm{d}\left(\boldsymbol{\nu}_{n}\right)_{t}(x, \theta, F)-\left\langle\boldsymbol{l}\left(\tau^{n}(t)\right), \boldsymbol{v}_{n}(t)\right\rangle+\operatorname{Var}_{H}\left(\boldsymbol{\mu}_{n} ; 0, t\right) \leq \\
& \mathcal{W}\left(z_{0}, v_{0}\right)-\left\langle\boldsymbol{l}(0), v_{0}\right\rangle+\int_{0}^{\tau^{n}(t)}\left\langle\boldsymbol{\sigma}_{n}(s), \nabla \dot{\boldsymbol{\varphi}}(s)\right\rangle \mathrm{d} s-\int_{0}^{\tau^{n}(t)}\left[\left\langle\dot{\boldsymbol{l}}(s), \boldsymbol{v}_{n}(s)\right\rangle+\langle\boldsymbol{l}(s), \dot{\boldsymbol{\varphi}}(s)\rangle\right] \mathrm{d} s+\rho_{n}
\end{aligned}
$$

where $\rho_{n} \rightarrow 0$ as $n \rightarrow \infty$.

Proof. Thanks to (7.11) it is enough to prove that

$$
\rho_{n}^{1}:=\int_{0}^{\tau^{n}(t)}\left(\int_{D \times \mathbb{R}^{m} \times \mathbb{R}^{N \times d}}\left[\frac{\partial W}{\partial F}\left(\theta, F+\varepsilon^{n}(s, x)\right)-\frac{\partial W}{\partial F}(\theta, F)\right] \nabla \dot{\varphi}(s, x) \mathrm{d}\left(\boldsymbol{\nu}_{n}\right)_{s}(x, \theta, F)\right) \mathrm{d} s
$$

and

$$
\rho_{n}^{2}:=\int_{0}^{\tau^{n}(t)}\left\langle\dot{\boldsymbol{l}}(s), \boldsymbol{\varphi}\left(\tau^{n}(s)\right)-\boldsymbol{\varphi}(s)\right\rangle \mathrm{d} s
$$

tend to 0 as $n \rightarrow \infty$. Since $\varphi$ is uniformly continuous on $[0, T]$ with values in $\mathrm{H}^{1}\left(D ; \mathbb{R}^{N}\right)$, it is immediate to see that $\rho_{n}^{2} \rightarrow 0$ as $n \rightarrow \infty$. It remains to prove that, fixed $\delta>0, \rho_{n}^{1}<\delta$ for $n$ sufficiently large.

We recall that, since $\nabla \dot{\varphi} \in \mathrm{L}^{1}\left([0, T] ; \mathrm{L}^{2}\left(D ; \mathbb{R}^{N \times d}\right)\right)$, we can find a sequence $v_{j} \in \mathrm{C}^{0}\left([0, T] ; \mathrm{C}^{0}\left(\bar{D} ; \mathbb{R}^{N}\right)\right)$ such that $\int_{0}^{T}\left\|v_{j}(t)-\nabla \dot{\varphi}(t)\right\|_{2} \mathrm{~d} t \rightarrow 0$, as $j \rightarrow \infty$.

Since $\pi_{D}\left(\left(\boldsymbol{\nu}_{n}\right)_{s}\right)=\mathcal{L}^{d}$, using (W.2), we can deduce for every $M>1$ and every $s \in[0, T]$

$$
\begin{gathered}
\quad \int_{\{(x, \theta, F):|\theta|+|F|>M\}}\left[\frac{\partial W}{\partial F}\left(\theta, F+\varepsilon^{n}(s, x)\right)-\frac{\partial W}{\partial F}(\theta, F)\right] \nabla \dot{\boldsymbol{\varphi}}(s) \mathrm{d}\left(\boldsymbol{\nu}_{n}\right)_{s}(x, \theta, F) \leq \\
C \int_{\{(x, \theta, F):|\theta|+|F|>M\}} 2(1+|\theta|+|F|)\left|\nabla \dot{\boldsymbol{\varphi}}(s, x)-v_{j}(s, x)\right| \mathrm{d}\left(\boldsymbol{\nu}_{n}\right)_{s}(x, \theta, F) \\
+C \int_{\{(x, \theta, F):|\theta|+|F|>M\}} 2(1+|\theta|+|F|)\left|v_{j}(s, x)\right| \mathrm{d}\left(\boldsymbol{\nu}_{n}\right)_{s}(x, \theta, F)+C\left\|\varepsilon^{n}(s)\right\|_{2}\|\nabla \dot{\boldsymbol{\varphi}}(s)\|_{2} ;
\end{gathered}
$$

therefore, thanks to Lemma 7.4, for every $j$ we have

$$
\begin{aligned}
& \int_{0}^{\tau^{n}(t)}\left(\int_{\{(x, \theta, F):|\theta|+|F|>M\}}\left[\frac{\partial W}{\partial F}\left(\theta, F+\varepsilon^{n}(s, x)\right)-\frac{\partial W}{\partial F}(\theta, F)\right] \nabla \dot{\boldsymbol{\varphi}}(s) \mathrm{d}\left(\boldsymbol{\nu}_{n}\right)_{s}(x, \theta, F)\right) \mathrm{d} s \leq \\
& 8 C\left[\sup _{s \in[0, T]} \sup _{n} \int_{D \times \mathbb{R}^{m} \times \mathbb{R}^{N \times d}}|(\theta, F)|^{2} \mathrm{~d}\left(\boldsymbol{\nu}_{n}\right)_{s}(x, \theta, F)\right]^{1 / 2} \int_{0}^{t}\left\|\nabla \dot{\boldsymbol{\varphi}}(s)-v_{j}(s)\right\|_{2} \mathrm{~d} s \\
+ & 4 T \sup _{s \in[0, T]} \sup _{n} \int_{D \times \mathbb{R}^{m} \times \mathbb{R}^{N \times d}}|(\theta, F)|^{2} \mathrm{~d}\left(\boldsymbol{\nu}_{n}\right)_{s}(x, \theta, F) \frac{\left\|v_{j}\right\|_{\infty}}{M}+C \sup _{s \in[0, T]}\left\|\varepsilon^{n}(s)\right\|_{2} \int_{0}^{T}\|\nabla \dot{\boldsymbol{\varphi}}(s)\|_{2} \mathrm{~d} s .
\end{aligned}
$$


Since $s \mapsto \varphi(s)$ is continuous from $[0, T]$ into $\mathrm{H}^{1}\left(D ; \mathbb{R}^{N}\right)$, the term in the last line tends to 0 as $n \rightarrow \infty$. Therefore there exist $\bar{j}$ and $\bar{M}$ such that

$$
\int_{0}^{\tau^{n}(t)}\left(\int_{\{(x, \theta, F):|\theta|+|F|>\bar{M}\}}\left[\frac{\partial W}{\partial F}\left(\theta, F+\varepsilon^{n}(s, x)\right)-\frac{\partial W}{\partial F}(\theta, F)\right] \nabla \dot{\boldsymbol{\varphi}}(s) \mathrm{d}\left(\boldsymbol{\nu}_{n}\right)_{s}(x, \theta, F)\right) \mathrm{d} s \leq \frac{\delta}{2},
$$

for $n$ sufficiently large.

We now consider the contribution of the integral on $\{(x, \theta, F):|\theta|+|F| \leq \bar{M}\}$.

For every $M>0$ and $r>0$, define

$$
\omega_{M}(r):=\sup _{|(\theta, F)| \leq M,\left|\left(\theta^{\prime}, F^{\prime}\right)\right| \leq r}\left|\frac{\partial W}{\partial F}\left(\theta+\theta^{\prime}, F+F^{\prime}\right)-\frac{\partial W}{\partial F}(\theta, F)\right| .
$$

Thanks to the continuity properties of $\frac{\partial W}{\partial F}$, for every $M$ we have $\omega_{M}(r) \rightarrow 0$ as $r$ tends to 0 ; moreover it is $\omega_{M}(r) \leq 2 C(M+1)+C r$.

In particular, for $\bar{M}$ chosen before, it is immediate that

$$
\begin{aligned}
& \int_{0}^{\tau^{n}(t)}\left(\int_{\{(x, \theta, F):|\theta|+|F| \leq \bar{M}\}}\left[\frac{\partial W}{\partial F}\left(\theta, F+\varepsilon^{n}(s, x)\right)-\frac{\partial W}{\partial F}(\theta, F)\right] \nabla \dot{\boldsymbol{\varphi}}(s) \mathrm{d}\left(\boldsymbol{\nu}_{n}\right)_{s}(x, \theta, F)\right) \mathrm{d} s \leq \\
& \int_{0}^{t}\left(\int_{D} \omega_{\bar{M}}\left(\left|\varepsilon^{n}(s, x)\right|\right)|\nabla \dot{\varphi}(s, x)| \mathrm{d} x\right) \mathrm{d} s ;
\end{aligned}
$$

by the Dominated Convergence Theorem, we have

$$
\left\|\omega_{\bar{M}}\left(\left|\varepsilon^{n}(s)\right|\right)\right\|_{2} \rightarrow 0
$$

for a.e. $s \in[0, T]$, as $n$ tends to $\infty$; since we have the estimate

$$
\left\|\omega_{\bar{M}}\left(\left|\varepsilon^{n}(s)\right|\right)\right\|_{2}\|\nabla \dot{\varphi}(s)\|_{2} \leq C\|\nabla \dot{\varphi}(s)\|_{2}\left(2 \bar{M}+2+\left\|\varepsilon^{n}(s)\right\|_{2}\right),
$$

for every $s \in[0, T]$ and $\sup _{s \in[0, T]}\left\|\varepsilon^{n}(s)\right\|_{2} \rightarrow 0$ as $n \rightarrow \infty$, we can apply again the Dominated Convergence Theorem and obtain

$$
\int_{0}^{\tau^{n}(t)}\left(\int_{\{(x, \theta, F):|\theta|+|F| \leq \bar{M}\}}\left[\frac{\partial W}{\partial F}\left(\theta, F+\varepsilon^{n}(s, x)\right)-\frac{\partial W}{\partial F}(\theta, F)\right] \nabla \dot{\varphi}(s) \mathrm{d}\left(\boldsymbol{\nu}_{n}\right)_{s}(x, \theta, F)\right) \mathrm{d} s \leq \frac{\delta}{2}
$$

for $n$ sufficiently large. Therefore (7.16) and (7.17) give the thesis.

\subsection{Passage to the limit}

Thanks to (7.12), (7.13), and hypothesis (H.2), we can apply our version of Helly Theorem (Th. 4.10) to the sequence $\boldsymbol{\mu}_{n}$ and obtain a subsequence, still indicated by $\boldsymbol{\mu}_{n}$, a subset $\Theta$ of $[0, T]$, containing 0 , with $\mathcal{L}^{1}([0, T] \backslash \Theta)=0$, and $\boldsymbol{\mu} \in S Y_{-}^{2}\left([0, T], D ; \mathbb{R}^{m}\right)$, such that, for every finite sequence $t_{1}<\ldots<t_{l}$ in $\Theta$, we have

$$
\left(\boldsymbol{\mu}_{n}\right)_{t_{1} \ldots t_{l}} \rightarrow \boldsymbol{\mu}_{t_{1} \ldots t_{l}}, \quad 2 \text {-weakly* }
$$


For every $t \in \Theta$ we choose an increasing sequence of integers $n_{k}^{t}$, possibly depending on $t$, such that

$$
\underset{n}{\limsup }\left[\left\langle\boldsymbol{\sigma}_{n}(t), \nabla \dot{\boldsymbol{\varphi}}(t)\right\rangle+\left\langle\dot{\boldsymbol{l}}(t), \boldsymbol{v}_{n}(t)\right\rangle\right]=\lim _{k}\left[\left\langle\boldsymbol{\sigma}_{n_{k}^{t}}(t), \nabla \dot{\boldsymbol{\varphi}}(t)\right\rangle+\left\langle\dot{\boldsymbol{l}}(t), \boldsymbol{v}_{n_{k}^{t}}(t)\right\rangle\right],
$$

where $\boldsymbol{v}_{n}(t)$ is defined as in Remark 6.8 (this choice is crucial in order to apply the argument in [4], Sect. 7). Thanks to (7.12) and Lemma 4.13, there exists $\boldsymbol{\nu} \in Y^{2}\left(D ; \mathbb{R}^{m} \times \mathbb{R}^{N \times d}\right)^{[0, T]}$, such that $\tilde{\pi}_{\mathbb{R}^{m}}\left(\boldsymbol{\nu}_{t}\right)=\boldsymbol{\mu}_{t}$ for every $t \in[0, T]$, and satisfying the following properties:

(conv) For every $t \in \Theta$, there exists a subsequence of $\left(\boldsymbol{\nu}_{n_{k}^{t}}\right)_{k}$, still denoted by $\left(\boldsymbol{\nu}_{n_{k}^{t}}\right)_{k}$, such that

$$
\left(\boldsymbol{\nu}_{n_{k}^{t}}\right)_{t} \rightarrow \boldsymbol{\nu}_{t}, \quad 2 \text {-weakly*. }
$$

(appr) For every $t \in[0, T] \backslash \Theta$, there exists a sequence $s^{j} \in \Theta$, converging to $t$, with $s^{j} \leq t$, such that

$$
\boldsymbol{\nu}_{s^{j}} \rightarrow \boldsymbol{\nu}_{t}, \quad 2 \text {-weakly*. }
$$

Note that the map (6.23) is measurable on $[0, T]$ since

$$
\langle\boldsymbol{\sigma}(t), \nabla \dot{\boldsymbol{\varphi}}(t)\rangle-\langle\dot{\boldsymbol{l}}(t), \boldsymbol{v}(t)\rangle=\underset{n}{\limsup }\left[\left\langle\boldsymbol{\sigma}_{n}(t), \nabla \dot{\boldsymbol{\varphi}}(t)\right\rangle-\left\langle\dot{\boldsymbol{l}}(t), \boldsymbol{v}_{n}(t)\right\rangle\right],
$$

for every $t \in \Theta$, thanks to (7.19), (W.2), Remarks 4.3 and 6.8 .

It can be shown that $(\boldsymbol{\nu}, \boldsymbol{\mu}) \in A Y([0, T], \boldsymbol{\varphi})$. Indeed, thanks to (7.18) and (7.20), we can apply Lemma 6.7 to get

$$
(\boldsymbol{\nu}, \boldsymbol{\mu}) \in A Y(\Theta, \varphi)
$$

Let now $t_{1}<\ldots<t_{l}$ be a finite sequence in $[0, T]$. Thanks to left continuity of $\boldsymbol{\mu}$ and to (appr), for every $i=1, \ldots, l$, there exists a sequence $s_{i}^{j} \rightarrow t_{i}$ as $j \rightarrow \infty$, with $s_{i}^{j} \leq t_{i}$, such that

$$
\begin{gathered}
\boldsymbol{\mu}_{s_{1}^{j} \ldots s_{n}^{j}} \rightarrow \boldsymbol{\mu}_{t_{1} \ldots t_{n}}, \quad 2 \text {-weakly* } \\
\boldsymbol{\nu}_{s_{i}^{j}} \rightarrow \boldsymbol{\nu}_{t_{i}}, \quad 2 \text {-weakly* for every } i=1, \ldots, n
\end{gathered}
$$

as $j \rightarrow \infty$. If we define $\varphi^{j}:\left\{t_{1}, \ldots, t_{l}\right\} \rightarrow \mathrm{H}^{1}\left(D ; \mathbb{R}^{N}\right)$ by $\varphi^{j}\left(t_{i}\right):=\varphi\left(s_{i}^{j}\right)$, for every $i=1, \ldots, n$, we have that $\varphi^{j}\left(t_{i}\right) \rightarrow \varphi\left(t_{i}\right)$ strongly in $\mathrm{H}^{1}\left(D ; \mathbb{R}^{N}\right)$, for every $i=1, \ldots, n$; if we define $\left(\tilde{\boldsymbol{\nu}}^{j}, \tilde{\boldsymbol{\mu}}^{j}\right) \in Y^{2}\left(D ; \mathbb{R}^{m} \times\right.$ $\left.\mathbb{R}^{N \times d}\right)^{\left\{t_{1}, \ldots, t_{l}\right\}} \times S Y^{2}\left(\left\{t_{1}, \ldots, t_{l}\right\}, D ; \mathbb{R}^{m}\right)$ by

$$
\begin{gathered}
\tilde{\boldsymbol{\nu}}_{t_{i}}^{j}:=\boldsymbol{\nu}_{s_{i}^{j}}, \quad \text { for every } i=1, \ldots, n, \\
\tilde{\boldsymbol{\mu}}_{t_{1} \ldots t_{l}}^{j}:=\boldsymbol{\mu}_{s_{1}^{j} \ldots s_{l}^{j}},
\end{gathered}
$$

thanks to (7.23), we have that $\left(\tilde{\boldsymbol{\nu}}^{j}, \tilde{\boldsymbol{\mu}}^{j}\right) \in A Y\left(\left\{t_{1}, \ldots, t_{n}\right\}, \varphi^{j}\right)$ and

$$
\begin{gathered}
\left(\tilde{\boldsymbol{\mu}}^{j}\right)_{t_{1} \ldots t_{l}} \rightarrow \boldsymbol{\mu}_{t_{1} \ldots t_{l}}, \quad 2 \text {-weakly* } \\
\left(\tilde{\boldsymbol{\nu}}^{j}\right)_{t_{i}} \rightarrow \boldsymbol{\nu}_{t_{i}}, \quad 2 \text {-weakly* for every } i=1, \ldots, l,
\end{gathered}
$$

as $j \rightarrow \infty$. Hence we are again in the hypotheses of Lemma 6.7 and we can conclude that $(\boldsymbol{\nu}, \boldsymbol{\mu}) \in A Y([0, T], \boldsymbol{\varphi})$.

By construction, $(\boldsymbol{\nu}, \boldsymbol{\mu})$ satisfies (ev0).

Now we want to prove that $(\boldsymbol{\nu}, \boldsymbol{\mu})$ satisfies (ev1). 
Let $\tilde{z} \in \mathrm{L}^{2}\left(D ; \mathbb{R}^{m}\right), \tilde{u} \in \mathrm{H}_{\Gamma_{0}}^{1}(0)$; for every $n$ and for every $i=1, \ldots, k(n)$, let consider the pair $(\hat{\boldsymbol{\nu}}, \hat{\boldsymbol{\mu}})$, where $\hat{\boldsymbol{\nu}}:=\left(\left(\boldsymbol{\nu}_{n}^{i}\right)_{t_{n}^{0}}, \ldots,\left(\boldsymbol{\nu}_{n}^{i}\right)_{t_{n}^{i-1}}, \mathcal{T}_{(\tilde{z}, \nabla \tilde{u})}\left(\left(\boldsymbol{\nu}_{n}^{i}\right)_{t_{n}^{i}}\right)\right)$ and $\hat{\boldsymbol{\mu}}$ is the unique compatible system in $S Y^{2}\left(\left\{t_{n}^{0}, \ldots, t_{n}^{i}\right\}, D ; \mathbb{R}^{m}\right)$ satisfying $\hat{\boldsymbol{\mu}}_{t_{n}^{0} \ldots t_{n}^{i}}=\tilde{\mathcal{T}}_{\tilde{z}}^{i+1}\left(\left(\boldsymbol{\mu}_{n}^{i}\right)_{t_{n}^{0}} \ldots t_{n}^{i}\right)$, with

$$
\mathcal{T}_{(\tilde{z}, \nabla \tilde{u})}(x, \theta, F)=(x, \theta+\tilde{z}(x), F+\nabla \tilde{u}(x))
$$

and

$$
\tilde{\mathcal{T}}_{\tilde{z}}^{i+1}\left(x, \theta_{0}, \ldots, \theta_{i}\right)=\left(x, \theta_{0}, \ldots, \theta_{i-1}, \theta_{i}+\tilde{z}(x)\right) .
$$

This is an element of $A Y\left(\left\{t_{n}^{0}, \ldots, t_{n}^{i}\right\}, \varphi\right)$ and satisfies (7.2) and (7.3), hence we can use it as a competitor to obtain

$$
\begin{gathered}
\int_{D \times \mathbb{R}^{m} \times \mathbb{R}^{N \times d}} W(\theta, F) \mathrm{d}\left(\boldsymbol{\nu}_{n}^{i}\right)_{t_{n}^{i}}(x, \theta, F)-\left\langle\boldsymbol{l}_{n}^{i}, \boldsymbol{v}_{n}^{i}\left(t_{n}^{i}\right)\right\rangle+\int_{D \times\left(\mathbb{R}^{m}\right)^{i+1}} H\left(\theta_{i}-\theta_{i-1}\right) \mathrm{d}\left(\boldsymbol{\mu}_{n}^{i}\right)_{t_{n}^{0} \ldots t_{n}^{i}} \\
\leq \int_{D \times \mathbb{R}^{m} \times \mathbb{R}^{N \times d}} W(\theta+\tilde{z}(x), F+\nabla \tilde{u}(x)) \mathrm{d}\left(\boldsymbol{\nu}_{n}^{i}\right)_{t_{n}^{i}}(x, \theta, F)-\left\langle\boldsymbol{l}_{n}^{i}, \boldsymbol{v}_{n}^{i}\left(t_{n}^{i}\right)+\tilde{u}\right\rangle \\
+\int_{D \times\left(\mathbb{R}^{m}\right)^{i+1}} H\left(\theta_{i}+\tilde{z}(x)-\theta_{i-1}\right) \mathrm{d}\left(\boldsymbol{\mu}_{n}^{i}\right)_{t_{n}^{0} \ldots t_{n}^{i} .}
\end{gathered}
$$

Thanks to the triangular inequality for $H$ (which follows from (H.1)), this implies that

$$
\int_{D \times \mathbb{R}^{m} \times \mathbb{R}^{N \times d}}[W(\theta, F)-W(\theta+\tilde{z}(x), F+\nabla \tilde{u}(x))] \mathrm{d}\left(\boldsymbol{\nu}_{n}^{i}\right)_{t_{n}^{i}}(x, \theta, F) \leq \mathcal{H}(\tilde{z})-\left\langle\boldsymbol{l}_{n}^{i}, \tilde{u}\right\rangle .
$$

By definition of $\boldsymbol{\nu}_{n}$, for every $t \in \Theta$ we obtain

$$
\int_{D \times \mathbb{R}^{m} \times \mathbb{R}^{N \times d}}[W(\theta, F)-W(\theta+\tilde{z}(x), F+\nabla \tilde{u}(x))] \mathrm{d}\left(\boldsymbol{\nu}_{n}\right)_{t}(x, \theta, F) \leq \mathcal{H}(\tilde{z})-\left\langle\boldsymbol{l}\left(\tau^{n}(t)\right), \tilde{u}\right\rangle .
$$

Since

$$
|W(\theta, F)-W(\theta+\tilde{z}(x), F+\nabla \tilde{u}(x))| \leq \tilde{C}\left[(|\tilde{z}(x)|+|\nabla \tilde{u}(x)|)^{2}+1\right]+\tilde{C}(|\tilde{z}(x)|+|\nabla \tilde{u}(x)|)(|\theta|+|F|),
$$

for a suitable positive constant $\tilde{C}$, and $\left(\boldsymbol{\nu}_{n_{k}^{t}}\right)_{t} \rightarrow \boldsymbol{\nu}_{t} 2$-weakly*, for a suitable subsequence $\left(\left(\boldsymbol{\nu}_{n_{k}^{t}}\right)_{t}\right)_{k}$, we can deduce from Remark 4.3 that

$$
\begin{aligned}
& \int_{D \times \mathbb{R}^{m} \times \mathbb{R}^{N \times d}}[W(\theta, F)-W(\theta+\tilde{z}(x), F+\nabla \tilde{u}(x))] \mathrm{d}\left(\boldsymbol{\nu}_{n_{k}^{t}}\right)_{t}(x, \theta, F) \rightarrow \\
& \int_{D \times \mathbb{R}^{m} \times \mathbb{R}^{N \times d}}[W(\theta, F)-W(\theta+\tilde{z}(x), F+\nabla \tilde{u}(x))] \mathrm{d} \boldsymbol{\nu}_{t}(x, \theta, F),
\end{aligned}
$$

as $n \rightarrow \infty$. Since $\boldsymbol{l}\left(\tau^{n}(t)\right) \rightarrow \boldsymbol{l}(t)$, strongly in $\mathrm{H}^{1}\left(D ; \mathbb{R}^{N}\right)^{*}$, for every $t \in[0, T]$, we obtain (ev1) for $t \in \Theta$.

Let now $t \in[0, T] \backslash \Theta$. Thanks to (appr), we can find a sequence $s^{j} \rightarrow t$, with $s^{j} \leq t$ and $s^{j} \in \Theta$, such that $\boldsymbol{\nu}_{s^{j}} \rightarrow \boldsymbol{\nu}_{t} 2$-weakly*; as before we have that

$$
\begin{aligned}
\int_{D \times \mathbb{R}^{m} \times \mathbb{R}^{N \times d}}[W(\theta, F)-W(\theta+\tilde{z}(x), F+\nabla \tilde{u}(x))] \mathrm{d} \boldsymbol{\nu}_{s^{j}}(x, \theta, F) \rightarrow \\
\int_{D \times \mathbb{R}^{m} \times \mathbb{R}^{N \times d}}[W(\theta, F)-W(\theta+\tilde{z}(x), F+\nabla \tilde{u}(x))] \mathrm{d} \boldsymbol{\nu}_{t}(x, \theta, F),
\end{aligned}
$$


as $j \rightarrow \infty$. Again, since $\boldsymbol{l}\left(s^{j}\right) \rightarrow \boldsymbol{l}(t)$ strongly in $\mathrm{H}^{1}\left(D ; \mathbb{R}^{N}\right)^{*}$, using (ev1) for $s^{j}$, we obtain (ev1) for every $t \in[0, T]$.

Now we want to prove (ev2). Fix $t \in \Theta$ and let $\left(\boldsymbol{\nu}_{n_{k}^{t}}\right)_{t}$ be a subsequence satisfying (7.19) and such that $\left(\boldsymbol{\nu}_{n_{k}^{t}}\right)_{t} \rightarrow \boldsymbol{\nu}_{t} 2$-weakly* as $k \rightarrow \infty$; then, thanks to (7.18),

$$
\mu_{n_{k}^{t}} \rightarrow \mu
$$

weakly* in $S Y^{2}\left([0, T], D ; \mathbb{R}^{m}\right)$; since the term containing $\boldsymbol{l}$ is continuous with respect to 2 -weak* convergence, the term containing $W$ is weakly lower semicontinuous and the variation is weakly lower semicontinuous thanks to (7.26) and Lemma 4.11, we have

$$
\begin{aligned}
\int_{D \times \mathbb{R}^{m} \times \mathbb{R}^{N \times d}} W(\theta, F) \mathrm{d} \boldsymbol{\nu}_{t}(x, \theta, F)-\langle\boldsymbol{l}(t), \boldsymbol{v}(t)\rangle+\operatorname{Var}_{H}(\boldsymbol{\mu} ; 0, t) \leq \\
\liminf _{k}\left[\int_{D \times \mathbb{R}^{m} \times \mathbb{R}^{N \times d}} W(\theta, F) \mathrm{d}\left(\boldsymbol{\nu}_{n_{t}^{k}}\right) t(x, \theta, F)-\left\langle\boldsymbol{l}\left(\tau^{n_{k}^{t}}(t)\right), \boldsymbol{v}_{n_{k}^{t}}(t)\right\rangle+\operatorname{Var}_{H}\left(\boldsymbol{\mu}_{n_{k}^{t}} ; 0, t\right)\right] .
\end{aligned}
$$

Using (7.15) of Lemma 7.5, we can deduce that

$$
\begin{gathered}
\int_{D \times \mathbb{R}^{m} \times \mathbb{R}^{N \times d}} W(\theta, F) \mathrm{d} \boldsymbol{\nu}_{t}(x, \theta, F)-\langle\boldsymbol{l}(t), \boldsymbol{v}(t)\rangle+\operatorname{Var}_{H}(\boldsymbol{\mu} ; 0, t) \leq \\
\mathcal{W}\left(z_{0}, v_{0}\right)-\langle\boldsymbol{l}(0), \boldsymbol{v}(0)\rangle-\int_{0}^{t}\langle\boldsymbol{l}(s), \dot{\boldsymbol{\varphi}}(s)\rangle \mathrm{d} s \\
+\liminf _{k} \int_{0}^{\tau^{n_{k}^{t}}(t)}\left[\left\langle\boldsymbol{\sigma}_{n_{k}^{t}}(s), \nabla \dot{\boldsymbol{\varphi}}(s)\right\rangle-\left\langle\dot{\boldsymbol{l}}(s), \boldsymbol{v}_{n_{k}^{t}}(s)\right\rangle\right] \mathrm{d} s .
\end{gathered}
$$

Since $\sup _{s \in[0, T]} \sup _{n}\left\|\boldsymbol{\sigma}_{n}(s)\right\|_{2}$ and $\sup _{s \in[0, T]} \sup _{n}\left\|\boldsymbol{v}_{n}(s)\right\|_{2}$ are finite, we can deduce, using Fatou Lemma, that

$$
\begin{aligned}
\liminf _{k} \int_{0}^{\tau^{n_{k}^{t}(t)}}\left[\left\langle\boldsymbol{\sigma}_{n_{k}^{t}}(s), \nabla \dot{\boldsymbol{\varphi}}(s)\right\rangle-\left\langle\dot{\boldsymbol{i}}(s), \boldsymbol{v}_{n_{k}^{t}}(s)\right\rangle\right] \mathrm{d} s & \leq \underset{n}{\limsup } \int_{0}^{\tau^{n}(t)}\left[\left\langle\boldsymbol{\sigma}_{n}(s), \nabla \dot{\boldsymbol{\varphi}}(s)\right\rangle-\left\langle\dot{\boldsymbol{l}}(s), \boldsymbol{v}_{n}(s)\right\rangle\right] \mathrm{d} s \\
& \leq \int_{0}^{t} \limsup _{n}\left[\left\langle\boldsymbol{\sigma}_{n}(s), \nabla \dot{\boldsymbol{\varphi}}(s)\right\rangle-\left\langle\dot{\boldsymbol{l}}(s), \boldsymbol{v}_{n}(s)\right\rangle\right] \mathrm{d} s .
\end{aligned}
$$

Thanks to (7.22) this implies that

$$
\liminf _{k} \int_{0}^{\tau^{n_{k}^{t}}(t)}\left[\left\langle\boldsymbol{\sigma}_{n_{k}^{t}}(s), \nabla \dot{\boldsymbol{\varphi}}(s)\right\rangle-\left\langle\dot{\boldsymbol{l}}(s), \boldsymbol{v}_{n_{k}^{t}}(s)\right\rangle\right] \mathrm{d} s \leq \int_{0}^{t}[\langle\boldsymbol{\sigma}(s), \nabla \dot{\boldsymbol{\varphi}}(s)\rangle-\langle\dot{\boldsymbol{l}}(s), \boldsymbol{v}(s)\rangle] \mathrm{d} s .
$$

This implies (ev2) for $t \in \Theta$.

Let now $t \in[0, T] \backslash \Theta$ and let $s^{j} \rightarrow t$, satisfy $s_{j} \leq t$, and (7.21); it is easy to verify, using Lemma 4.11, that

$$
\operatorname{Var}_{H}(\boldsymbol{\mu} ; 0, t) \leq \liminf _{j} \operatorname{Var}_{H}\left(\boldsymbol{\mu} ; 0, s^{j}\right),
$$

hence (ev2) for $t$ can be deduced from (ev2) for $s^{j}$.

\section{An alternative Proof of the eXISTEnCE RESUlt in THE QUASICONVEX CASE}

In this section we focus on the particular case of $W(\theta, \cdot)$ quasiconvex, which can be treated using a spatial regularization depending on the gradient of the internal variable, as proposed in [8]. 
Definition 8.1. Let $\eta>0, \varphi \in A C\left([0, T] ; \mathrm{H}^{1}\left(D ; \mathbb{R}^{N}\right)\right), \boldsymbol{l} \in A C\left([0, T] ; \mathrm{H}^{1}\left(D ; \mathbb{R}^{N}\right)^{*}\right), z_{0} \in \mathrm{H}^{1}\left(D ; \mathbb{R}^{m}\right)$, $v_{0} \in \mathcal{A}(0)$, and $T>0$. A solution of the $\eta$-regularized problem with external load $\boldsymbol{l}$, boundary datum $\boldsymbol{\varphi}$, and initial condition $\left(z_{0}, v_{0}\right)$, in the time interval $(0, T]$, is a pair $\left(\boldsymbol{z}_{\eta}, \boldsymbol{v}_{\eta}\right)$, with $\boldsymbol{z}_{\eta}:[0, T] \rightarrow \mathrm{H}^{1}\left(D ; \mathbb{R}^{m}\right)$ and $\boldsymbol{v}_{\eta}:[0, T] \rightarrow \mathrm{H}^{1}\left(D ; \mathbb{R}^{N}\right)$, satisfying the following properties:

$(\mathrm{ev} 0)_{\mathrm{reg}}$ initial condition: $\left(\boldsymbol{z}_{\eta}(0), \boldsymbol{v}_{\eta}(0)\right)=\left(z_{0}, v_{0}\right)$;

(ev1) reg kinematic admissibility: $\boldsymbol{v}_{\eta}(t) \in \mathcal{A}(t)$ for every $t \in[0, T]$;

(ev2) reg global stability: for every $t \in(0, T]$ and $(\hat{z}, \hat{v}) \in \mathrm{H}^{1}\left(D ; \mathbb{R}^{m}\right) \times \mathcal{A}(t)$

$$
\mathcal{W}\left(\boldsymbol{z}_{\eta}(t), \boldsymbol{v}_{\eta}(t)\right)-\left\langle\boldsymbol{l}(t), \boldsymbol{v}_{\eta}(t)\right\rangle+\frac{\eta}{2}\left\|\nabla \boldsymbol{z}_{\eta}(t)\right\|_{2}^{2} \leq \mathcal{W}(\hat{z}, \hat{v})-\langle\boldsymbol{l}(t), \hat{v}\rangle+\frac{\eta}{2}\|\nabla \hat{z}\|_{2}^{2}+\mathcal{H}\left(\hat{z}-\boldsymbol{z}_{\eta}(t)\right)
$$

(ev3) $)_{\mathrm{reg}}$ energy inequality: $\operatorname{Var}_{H}\left(\boldsymbol{z}_{\eta} ; 0, T\right)<\infty$, the map $t \mapsto\left[\left\langle\boldsymbol{\sigma}_{\eta}(t), \nabla \dot{\boldsymbol{\varphi}}(t)\right\rangle-\left\langle\dot{\boldsymbol{l}}(t), \boldsymbol{v}_{\eta}(t)\right\rangle\right]$ is measurable on $[0, T]$, and for every $t \in[0, T]$

$$
\begin{aligned}
\mathcal{W}\left(\boldsymbol{z}_{\eta}(t), \boldsymbol{v}_{\eta}(t)\right)- & \left\langle\boldsymbol{l}(t), \boldsymbol{v}_{\eta}(t)\right\rangle+\frac{\eta}{2}\left\|\nabla \boldsymbol{z}_{\eta}(t)\right\|_{2}^{2}+\operatorname{Var}_{H}\left(\boldsymbol{z}_{\eta} ; 0, t\right) \leq \\
& \mathcal{W}\left(z_{0}, v_{0}\right)-\left\langle\boldsymbol{l}(0), v_{0}\right\rangle+\frac{\eta}{2}\left\|\nabla z_{0}\right\|_{2}^{2}-\int_{0}^{t}\langle\boldsymbol{l}(s), \dot{\boldsymbol{\varphi}}(s)\rangle \mathrm{d} s+\int_{0}^{t}\left[\left\langle\boldsymbol{\sigma}_{\eta}(s), \nabla \dot{\boldsymbol{\varphi}}(s)\right\rangle-\left\langle\dot{\boldsymbol{l}}(s), \boldsymbol{v}_{\eta}(s)\right\rangle\right] \mathrm{d} s,
\end{aligned}
$$

where $\boldsymbol{\sigma}_{\eta}(s):=\frac{\partial W}{\partial F}\left(\boldsymbol{z}_{\eta}(s), \nabla \boldsymbol{v}_{\eta}(s)\right)$ for every $s \in[0, T]$.

The proof of Theorem 4.1 in [8] can be repeated in our case to obtain the following result.

Theorem 8.2. Let $\eta, \varphi, l,\left(z_{0}, v_{0}\right)$, and $T$ be as in Definition 8.1. Then there exists a solution of the $\eta$-regularized problem with external load $\boldsymbol{l}$, boundary datum $\boldsymbol{\varphi}$, and initial condition $\left(z_{0}, v_{0}\right)$, in the time interval $(0, T]$. Moreover there exists a positive constant $K_{\eta}$ such that

$$
\begin{aligned}
& \sup _{t \in[0, T]}\left\|\boldsymbol{z}_{\eta}(t)\right\|_{2} \leq K_{\eta}, \\
& \sup _{t \in[0, T]}\left\|\boldsymbol{v}_{\eta}(t)\right\|_{\mathrm{H}^{1}} \leq K_{\eta} .
\end{aligned}
$$

Lemma 8.3. Let $\boldsymbol{\varphi}, \boldsymbol{l}, v_{0}$, and $T$ be as in Definition 8.1, and let $\left(z_{0}^{\eta}\right)_{\eta>0}$ be a family of functions in $\mathrm{H}^{1}\left(D ; \mathbb{R}^{m}\right)$, such that

$$
\begin{gathered}
\sup _{\eta}\left\|z_{0}^{\eta}\right\|_{2} \leq M, \\
\sup _{\eta} \eta\left\|\nabla z_{0}^{\eta}\right\|_{2}^{2} \leq M,
\end{gathered}
$$

for a suitable positive constant $M$. Then there exists a positive constant $K$ such that the solutions $\left(\boldsymbol{z}_{\eta}, \boldsymbol{v}_{\eta}\right)$ of the $\eta$-regularized problems with initial condition $\left(z_{0}^{\eta}, v_{0}\right)$ satisfy the following conditions

$$
\begin{gathered}
\sup _{t \in[0, T]} \sup _{\eta}\left\|\boldsymbol{z}_{\eta}(t)\right\|_{2} \leq K, \quad \sup _{t \in[0, T]} \sup _{\eta}\left\|\nabla \boldsymbol{v}_{\eta}(t)\right\|_{2} \leq K, \\
\sup _{\eta} \operatorname{Var}_{H}\left(\boldsymbol{z}_{\eta} ; 0, T\right) \leq K, \quad \sup _{t \in[0, T]} \frac{\eta}{2}\left\|\nabla \boldsymbol{z}_{\eta}(t)\right\|_{2}^{2} \leq K .
\end{gathered}
$$

Proof. Using the fact that $\sup _{t \in[0, T]}\|\boldsymbol{l}(t)\|_{\left(\mathrm{H}^{1}\right)^{*}}, \int_{0}^{T}\|\dot{\boldsymbol{i}}(t)\|_{\left(\mathrm{H}^{1}\right)^{*}} \mathrm{~d} t$, and $\int_{0}^{T}\|\dot{\boldsymbol{\varphi}}(t)\|_{\mathrm{H}^{1}} \mathrm{~d} t$ are finite, the hypotheses on $W$, the hypotheses (8.3) and (8.4), and the inequalities (8.1) and (8.2), we can deduce from (ev3) reg that, for every $\eta>0$,

$$
c\left(\left\|\boldsymbol{z}_{\eta}(t)\right\|_{2}^{2}+\left\|\nabla \boldsymbol{v}_{\eta}(t)\right\|_{2}^{2}\right)-C \leq \tilde{C}+\tilde{c} \sup _{s \in[0, T]}\left(\left\|\boldsymbol{z}_{\eta}(s)\right\|_{2}+\left\|\boldsymbol{v}_{\eta}(s)\right\|_{\mathrm{H}^{1}}\right)
$$


for suitable positive constants $\tilde{c}, \tilde{C}$ independent of $t$ and $\eta$. Since this can be repeated for every $t \in[0, T]$, Poincaré inequality implies (8.5). Inequalities (8.6) come now from (ev3) reg and (8.5).

Remark 8.4. From (8.6) we can deduce that

$$
\eta_{n} \nabla \boldsymbol{z}_{\eta_{n}}(t) \rightarrow 0
$$

strongly in $\mathrm{L}^{2}\left(D ; \mathbb{R}^{m \times d}\right)$, for every positive sequence $\eta_{n} \rightarrow 0$ and every $t \in[0, T]$.

Definition 8.5. Given an external load $\boldsymbol{l} \in A C\left([0, T] ; \mathrm{H}^{1}\left(D ; \mathbb{R}^{N}\right)^{*}\right)$, an initial condition $\left(z_{0}, v_{0}\right) \in \mathrm{L}^{2}\left(D ; \mathbb{R}^{m}\right) \times$ $\mathcal{A}(0)$, a boundary datum $\varphi \in A C\left([0, T] ; \mathrm{H}^{1}\left(D ; \mathbb{R}^{N}\right)\right)$, and $T>0$, a quasistatic evolution obtained by spatial regularizations in the time interval $[0, T]$ is a pair $(\boldsymbol{\nu}, \boldsymbol{\mu}) \in Y^{2}\left(D ; \mathbb{R}^{m} \times \mathbb{R}^{N \times d}\right)^{[0, T]} \times S Y_{-}^{2}\left([0, T], D ; \mathbb{R}^{m}\right)$ with

$$
\tilde{\pi}_{\mathbb{R}^{m}}\left(\boldsymbol{\nu}_{t}\right)=\boldsymbol{\mu}_{t}, \quad \text { for every } t \in[0, T],
$$

for which there exist:

- a sequence $\left(z_{0}^{n}\right)_{n} \subset \mathrm{H}^{1}\left(D ; \mathbb{R}^{m}\right)$, with

$$
z_{0}^{n} \rightarrow z_{0} \quad \text { strongly in } \mathrm{L}^{2}\left(D ; \mathbb{R}^{m}\right),
$$

- a positive sequence $\eta_{n}$ converging to 0 , with

$$
\eta_{n}\left\|\nabla z_{0}^{n}\right\|_{2}^{2} \rightarrow 0
$$

- a subset $\Theta$ of $[0, T]$, with $0 \in \Theta$ and $\mathcal{L}^{1}([0, T] \backslash \Theta)=0$,

such that $\boldsymbol{\nu}$ and $\Theta$ satisfy property (appr) of Remark 6.16, and the solutions $\left(\boldsymbol{z}_{\eta_{n}}, \boldsymbol{v}_{\eta_{n}}\right)$ of the $\eta_{n}$-regularized problems with initial conditions $\left(z_{0}^{n}, v_{0}\right)$ in the time interval $(0, T]$ satisfy the following conditions:

(conv1)reg for every finite sequence $t_{1}<\ldots<t_{l}$ in $\Theta$

$$
\delta_{\left(\boldsymbol{z}_{\eta_{n}}\left(t_{1}\right), \ldots, \boldsymbol{z}_{\eta_{n}}\left(t_{l}\right)\right)} \rightarrow \boldsymbol{\mu}_{t_{1} \ldots t_{l}} \quad \text { 2-weakly* }
$$

as $n \rightarrow \infty$;

$(\operatorname{conv} 2)_{\text {reg }}$ for every $t \in \Theta$ there exists a subsequence $\left(\boldsymbol{z}_{\eta_{n_{k}^{t}}}, \boldsymbol{v}_{\eta_{n_{k}^{t}}}\right)_{k}$ of $\left(\boldsymbol{z}_{\eta_{n}}, \boldsymbol{v}_{\eta_{n}}\right)_{n}$, possibly depending on $t$, with

$$
\underset{n}{\limsup }\left[\left\langle\boldsymbol{\sigma}_{\eta_{n}}(t), \nabla \dot{\boldsymbol{\varphi}}(t)\right\rangle-\left\langle\dot{\boldsymbol{l}}(t), \boldsymbol{v}_{\eta_{n}}(t)\right\rangle\right]=\lim _{k}\left[\left\langle\boldsymbol{\sigma}_{\eta_{n_{k}^{t}}}(t), \nabla \dot{\boldsymbol{\varphi}}(t)\right\rangle-\left\langle\dot{\boldsymbol{l}}(t), \boldsymbol{v}_{\eta_{n_{k}^{t}}}(t)\right\rangle\right],
$$

and

$$
\left.\delta_{\left(\boldsymbol{z}_{\eta_{n_{k}^{t}}}\right.}(t), \nabla \boldsymbol{v}_{\eta_{n_{k}^{t}}}(t)\right) \rightarrow \boldsymbol{\nu}_{t} \quad \text { 2-weakly*, }
$$

as $k \rightarrow \infty$.

In the next theorem we will show that the quasistatic evolution obtained by spatial regularizations fulfills the requirements of Definition 6.14.

Theorem 8.6. Let $\boldsymbol{l} \in A C\left([0, T] ; \mathrm{H}^{1}\left(D ; \mathbb{R}^{N}\right)^{*}\right), \varphi \in A C\left([0, T] ; \mathrm{H}^{1}\left(D ; \mathbb{R}^{N}\right)\right),\left(z_{0}, v_{0}\right)$, and $T>0$ be as in Definition 8.5, and assume that

$$
\mathcal{W}\left(z_{0}, v_{0}\right) \leq \mathcal{W}(\hat{z}, \hat{v})-\left\langle\boldsymbol{l}(0), \hat{v}-v_{0}\right\rangle+\mathcal{H}\left(\hat{z}-z_{0}\right),
$$

for every $\hat{z} \in \mathrm{L}^{2}\left(D ; \mathbb{R}^{m}\right)$ and every $\hat{v} \in \mathcal{A}(0)$. Then a quasistatic evolution obtained by spatial regularizations in the time interval $[0, T]$ is a globally stable quasistatic evolution of Young measures with the same data in the time interval $[0, T]$. 
Proof. Let $(\boldsymbol{\nu}, \boldsymbol{\mu}) \in Y^{2}\left(D ; \mathbb{R}^{m} \times \mathbb{R}^{N \times d}\right)^{[0, T]} \times S Y_{-}^{2}\left([0, T], D ; \mathbb{R}^{m}\right)$ be a quasistatic evolution obtained by spatial regularizations.

First of all we show that $(\boldsymbol{\nu}, \boldsymbol{\mu}) \in A Y([0, T], \boldsymbol{\varphi})$ : thanks to (8.10) and (8.12), it is immediate to see that $(\boldsymbol{\nu}, \boldsymbol{\mu}) \in A Y(\Theta, \boldsymbol{\varphi})$; as in the proof of the main theorem, (appr) allows now to prove that $(\boldsymbol{\nu}, \boldsymbol{\mu}) \in A Y([0, T], \boldsymbol{\varphi})$. In particular, for every $t \in[0, T]$ there exists a function $\boldsymbol{v}(t) \in \mathcal{A}(t)$ such that $\nabla \boldsymbol{v}(t)=\operatorname{bar}\left(\tilde{\pi}_{\mathbb{R}^{N \times d}}\left(\boldsymbol{\nu}_{t}\right)\right)$, thanks to Remark 6.8 .

Let $\eta_{n}$ and $z_{0}^{n}$ be the sequences appearing in Definition 8.5, and let $\left(\boldsymbol{z}_{\eta_{n}}, \boldsymbol{v}_{\eta_{n}}\right)$ be the solutions of the $\eta_{n}$-regularized problems in the time interval $(0, T]$; (ev0) comes immediately from (ev0) reg, (8.8), and (8.12).

Now we prove that $(\boldsymbol{\nu}, \boldsymbol{\mu})$ satisfies (ev1).

For $t=0$ it comes immediately from (8.13).

For $t \neq 0$, we first show that (ev1) holds for test functions $\tilde{z} \in \mathrm{H}^{1}\left(D ; \mathbb{R}^{m}\right)$. Let $t \in \Theta \backslash 0,\left(\eta_{n_{k}^{t}}\right)_{k}$ be the sequence appearing in $(\operatorname{conv} 2)_{\mathrm{reg}}$, and $(\tilde{z}, \tilde{u}) \in \mathrm{H}^{1}\left(D ; \mathbb{R}^{m}\right) \times \mathrm{H}_{\Gamma_{0}}^{1}(0)$. By (ev1) reg, we have

$\mathcal{W}\left(\boldsymbol{z}_{\eta_{n_{k}^{t}}}(t), \boldsymbol{v}_{\eta_{n_{k}^{t}}}(t)\right)+\frac{\eta_{n_{k}^{t}}}{2}\left\|\nabla \boldsymbol{z}_{\eta_{n_{k}^{t}}}(t)\right\|_{2}^{2} \leq \mathcal{W}\left(\boldsymbol{z}_{\eta_{n_{k}^{t}}}(t)+\tilde{z}, \boldsymbol{v}_{\eta_{n_{k}^{t}}}(t)+\tilde{u}\right)-\langle\boldsymbol{l}(t), \tilde{u}\rangle+\frac{\eta_{n_{k}^{t}}}{2}\left\|\nabla \boldsymbol{z}_{\eta_{n_{k}^{t}}}(t)+\nabla \tilde{z}\right\|_{2}^{2}+\mathcal{H}(\tilde{z})$.

Thanks to Remark 8.4,

$$
\frac{\eta_{n_{k}^{t}}}{2}\left\|\nabla \boldsymbol{z}_{\eta_{n_{k}^{t}}}(t)+\nabla \tilde{z}\right\|_{2}^{2}-\frac{\eta_{n_{k}^{t}}}{2}\left\|\nabla \boldsymbol{z}_{\eta_{n_{k}^{t}}}(t)\right\|_{2}^{2}=\frac{\eta_{n_{k}^{t}}}{2}\|\nabla \tilde{z}\|_{2}^{2}+\left\langle\eta_{n_{k}^{t}} \nabla \boldsymbol{z}_{\eta_{n_{k}^{t}}}(t), \nabla \tilde{z}\right\rangle, \rightarrow 0,
$$

as $k \rightarrow \infty$. On the other hand we have

$$
\begin{aligned}
& \mathcal{W}\left(\boldsymbol{z}_{\eta_{n_{k}^{t}}}(t)+\tilde{z}, \boldsymbol{v}_{\eta_{n_{k}^{t}}}(t)+\tilde{u}\right)-\mathcal{W}\left(\boldsymbol{z}_{\eta_{n_{k}^{t}}}(t), \boldsymbol{v}_{\eta_{n_{k}^{t}}}(t)\right)= \\
& \qquad \int_{D \times \mathbb{R}^{m} \times \mathbb{R}^{N \times d}}[W(\theta+\tilde{z}(x), F+\nabla \tilde{u}(x))-W(\theta, F)] \mathrm{d} \delta_{\left(\boldsymbol{z}_{\eta_{n_{k}}}(t), \nabla \boldsymbol{v}_{\eta_{n_{k}^{t}}}(t)\right)}(x, \theta, F) ;
\end{aligned}
$$

since

$$
|W(\theta+\tilde{z}(x), F+\nabla \tilde{u}(x))-W(\theta, F)| \leq \tilde{C}\left[(|\tilde{z}(x)|+|\nabla \tilde{u}(x)|)^{2}+1\right]+\tilde{C}(|\tilde{z}(x)|+|\nabla \tilde{u}(x)|)(|\theta|+|F|),
$$

thanks to Remarks 4.3 and (8.12) we can deduce that

$$
\begin{aligned}
\int_{D \times \mathbb{R}^{m} \times \mathbb{R}^{N \times d}}[W(\theta+\tilde{z}(x), F+\nabla \tilde{u}(x))-W(\theta, F)] \mathrm{d} \delta_{\left(\boldsymbol{z}_{\eta_{n_{k}^{t}}}(t), \nabla \boldsymbol{v}_{\eta_{n_{k}^{t}}}(t)\right)}(x, \theta, F) \rightarrow \\
\int_{D \times \mathbb{R}^{m} \times \mathbb{R}^{N \times d}}[W(\theta+\tilde{z}(x), F+\nabla \tilde{u}(x))-W(\theta, F)] \mathrm{d} \boldsymbol{\nu}_{t}(x, \theta, F),
\end{aligned}
$$

and hence we can conclude that

$$
\int_{D \times \mathbb{R}^{m} \times \mathbb{R}^{N \times d}} W(\theta, F) \mathrm{d} \boldsymbol{\nu}_{t}(x, \theta, F) \leq \int_{D \times \mathbb{R}^{m} \times \mathbb{R}^{N \times d}} W(\theta+\tilde{z}(x), F+\nabla \tilde{u}(x)) \mathrm{d} \boldsymbol{\nu}_{t}(x, \theta, F)-\langle\boldsymbol{l}(t), \tilde{u}\rangle+\mathcal{H}(\tilde{z}) .
$$

Using (appr) it is easy to extend the previous inequality to $t \in[0, T] \backslash \Theta$ reasoning as in the proof of the main theorem.

Now we prove that (8.15) holds also for test functions $\tilde{z} \in \mathrm{L}^{2}\left(D ; \mathbb{R}^{m}\right)$. Let $t \in(0, T], \tilde{z} \in \mathrm{L}^{2}\left(D ; \mathbb{R}^{m}\right)$, $\tilde{u} \in \mathrm{H}_{\Gamma_{0}}^{1}(0)$, and assume that $\left(\tilde{z}_{h}\right)_{h}$ is a sequence in $\mathrm{H}^{1}\left(D ; \mathbb{R}^{m}\right)$ with $\tilde{z}_{h} \rightarrow \tilde{z}$ strongly in $\mathrm{L}^{2}\left(D ; \mathbb{R}^{m}\right)$; then we have

$$
\mathcal{H}\left(\tilde{z}_{h}\right) \rightarrow \mathcal{H}(\tilde{z}),
$$


and

$$
\int_{D \times \mathbb{R}^{m} \times \mathbb{R}^{N \times d}}\left[W(\theta+\tilde{z}(x), F+\nabla \tilde{u}(x))-W\left(\theta+\tilde{z}_{h}(x), F+\nabla \tilde{u}(x)\right)\right] \mathrm{d} \boldsymbol{\nu}_{t}(x, \theta, F) \rightarrow 0,
$$

thanks to the hypotheses on $H$, and to (W.2) and (2.2), respectively; hence (ev1) for test functions $\tilde{z}$ and $\tilde{u}$ can be deduce from (8.15) applied to $\tilde{z}_{h}$ and $\tilde{u}$.

Finally we prove (ev2).

Thanks to (8.8) and (8.9), we have

$$
\mathcal{W}\left(z_{0}^{n}, v_{0}\right)+\frac{\eta_{n}}{2}\left\|\nabla z_{0}^{n}\right\|_{2}^{2} \rightarrow \mathcal{W}\left(z_{0}, v_{0}\right)
$$

as $n \rightarrow \infty$; therefore we can argue as in the proof of the main theorem using (ev2) reg and the approximation properties of $(\boldsymbol{\nu}, \boldsymbol{\mu})$.

Theorem 8.7. Let $\boldsymbol{l} \in A C\left([0, T] ; \mathrm{H}^{1}\left(D ; \mathbb{R}^{N}\right)^{*}\right), \varphi \in A C\left([0, T] ; \mathrm{H}^{1}\left(D ; \mathbb{R}^{N}\right)\right), T>0$, and $\left(z_{0}, v_{0}\right) \in \mathrm{L}^{2}\left(D ; \mathbb{R}^{m}\right) \times$ $\mathcal{A}(0)$. Then there exists a quasistatic evolution obtained by spatial regularizations with external load $\boldsymbol{l}$, boundary datum $\varphi$, and initial condition $\left(z_{0}, v_{0}\right)$, in the time interval $[0, T]$.

Proof. Fix a sequence $\left(z_{0}^{n}\right)_{n}$ in $\mathrm{H}^{1}\left(D ; \mathbb{R}^{m}\right)$ satisfying (8.8) and a positive sequence $\eta_{n} \rightarrow 0$ satisfying (8.9); let $\left(\boldsymbol{z}_{\eta_{n}}, \boldsymbol{v}_{\eta_{n}}\right)$ be the solutions of the $\eta_{n}$-regularized problems with external load $\boldsymbol{l}$, boundary datum $\boldsymbol{\varphi}$, and initial condition $\left(z_{0}^{n}, v_{0}\right)$. Thanks to $(8.5),(8.6)$, and (H.2), we can apply Theorem 4.10 to obtain a subset $\Theta$ of $[0, T]$ with $0 \in \Theta$ and $\mathcal{L}^{1}([0, T] \backslash \Theta)=0$, a compatible system $\boldsymbol{\mu} \in S Y_{-}^{2}\left([0, T], D ; \mathbb{R}^{m}\right)$, and a subsequence still indicated by $\left(\eta_{n}\right)_{n}$ satisfying (conv1) reg. For every $t \in \Theta$ we select a subsequence $\left(\eta_{n_{k}^{t}}\right)_{k}$ of $\left(\eta_{n}\right)_{n}$, possibly depending on $t$, satisfying (8.11). Thanks to (8.5), we can apply Lemma 4.13 to obtain another subsequence still denoted by $\left(\eta_{n_{k}^{t}}\right)_{k}$ and $\boldsymbol{\nu} \in Y^{2}\left(D ; \mathbb{R}^{m} \times \mathbb{R}^{N \times d}\right)^{[0, T]}$ which verify conditions (conv2) reg, (appr), and (8.7).

Acknowledgements. I wish to thank Prof. Gianni Dal Maso for having proposed to me this problem, and for many helpful and interesting discussions.

\section{REFERENCES}

[1] E. Acerbi and N. Fusco, Semicontinuity problems in the calculus of variations. Arch. Rational Mech. Anal. 86 (1984) $125-145$.

[2] J.M. Ball, A version of the fundamental theorem for Young measures, in PDE's and continuum models of phase transitions (Nice, 1988), Lecture Notes in Physics, Springer-Verlag, Berlin (1989) 207-215.

[3] H. Brezis, Opérateurs maximaux monotones et semi-groupes de contractions dans les espaces de Hilbert. North-Holland, Amsterdam-London; American Elsevier, New York (1973).

[4] G. Dal Maso, G. Francfort and R. Toader, Quasistatic crack growth in nonlinear elasticity. Arch. Rational Mech. Anal. 176 (2005) 165-225.

[5] G. Dal Maso, A. De Simone, M.G. Mora and M. Morini, Time-dependent systems of generalized Young measures. Netw. Heterog. Media 2 (2007) 1-36.

[6] G. Dal Maso, A. De Simone, M.G. Mora and M. Morini, Globally stable quasistatic evolution in plasticity with softening. Netw. Heterog. Media (to appear).

[7] I. Fonseca, S. Müller and P. Pedregal, Analysis of concentration and oscillation effects generated by gradients. SIAM J. Math. Anal. 29 (1998) 736-756.

[8] G. Francfort and A. Mielke, Existence results for a class of rate-independent material models with nonconvex elastic energy. J. Reine Angew. Math. 595 (2006) 55-91.

[9] M. Kočvara, A. Mielke and T. Roubíček, A rate-independent approach to the delamination problem. Math. Mech. Solids 11 (2006) 423-447.

[10] A.N. Kolmogorov, Foundations of the Theory of Probability. Chelsea Publishing Company, 2nd edition, New York (1956).

[11] C. Miehe and M. Lambrecht, Analysis of microstructure development in shearbands by energy relaxation of incremental stress potentials: large-strain theory for standard dissipative solids. Internat. J. Numer. Methods Engrg. 58 (2003) 1-41.

[12] C. Miehe, J. Schotte and M. Lambrecht, Computational homogenization of materials with microstructures based on incremental variational formulations, in IUTAM Symposium on Computational Mechanics of Solid Materials at Large Strains (Stuttgart, 2001), Solid Mech. Appl., Kluwer Acad. Publ., Dordrecht (2003) 87-100. 
[13] A. Mielke, Evolution of rate-independent systems, in Evolutionary equations, Vol. II, C.M. Dafermos and E. Feireisl Eds., Handbook of Differential Equations, Elsevier/North-Holland, Amsterdam (2005) 461-559.

[14] A. Mielke and T. Roubíček, Rate-independent damage processes in nonlinear elasticity. Math. Models Methods Appl. Sci. 16 (2006) 177-209.

[15] A. Mielke, F. Theil and V.I. Levitas, A variational formulation of rate-independent phase transformations using an extremum principle. Arch. Rational Mech. Anal. 162 (2002) 137-177.

[16] M. Ortiz and E. Repetto, Nonconvex energy minimization and dislocation structures in ductile single crystals. J. Mech. Physics Solids 47 (1999) 397-462.

[17] P. Pedregal, Parametrized measures and variational principles. Progress in Nonlinear Differential Equations and their Applications 30. Birkhäuser Verlag, Basel (1997).

[18] M. Valadier, Young measures, in Methods of nonconvex analysis (Varenna, 1989), Lecture Notes in Mathematics, SpringerVerlag, Berlin (1990) 152-188. 